\title{
TWISTOR TRANSFORM OF VECTOR BUNDLES
}

\author{
STEPHEN A. HUGGETT and SERGEY A. MERKULOV
}

\section{Introduction.}

Let $\left\{X_{t} \hookrightarrow Y \mid t \in M\right\}$ be a family of compact complex submanifolds $X_{t}$ of a complex manifold $Y$ parametrised by a complex manifold $M$. In this paper we study vector bundles and linear connections on the moduli space $M$ induced from holomorphic vector bundles on the space $Y$. The origin of this approach lies in the Ward transform [W] of holomorphic vector bundles $E$ on $Y$ which are trivial when restricted to each submanifold $X_{t}$ of the family $\left\{X_{t} \hookrightarrow Y \mid t \in M\right\}$. We consider a more general class of holomorphic vector bundles $E$ on twistor spaces $Y$ which, when restricted to a submanifold $X_{t}$ of the family, have the same integer $h^{0}\left(X_{t},\left.E\right|_{X_{t}}\right)$ for all $t \in M$. With such a vector bundle $E$ on $Y$ there is an associated vector bundle $\mathcal{E}$ on $M$ whose fibre at $t \in M$ is isomorphic to $H^{0}\left(X_{t},\left.E\right|_{X_{t}}\right)$. It is shown that provided the cohomology groups $H^{0}\left(X_{t},\left.E\right|_{X_{t}} \otimes N_{t}^{*}\right)$ and $H^{1}\left(X_{t},\left.E\right|_{X_{t}} \otimes N_{t}^{*}\right)$ vanish (where $N_{t}$ is the normal bundle of $\left.X_{t} \hookrightarrow Y\right)$, the vector bundle $\mathcal{E}$ induced on the parameter space $M$ comes equipped with a distinguished linear connection $\nabla$ satisfying some natural integrability conditions (cf. [L,E,B-E]). The curvature tensor of $\nabla$ is represented, at each $t \in M$, by a cohomology class in $H^{1}\left(X_{t},\left.E\right|_{X_{t}} \otimes \odot^{2} N^{*}\right) \otimes\left(H^{0}\left(X_{t},\left.E\right|_{X_{t}}\right)\right)^{*}$. This theorem-construction gives a very simple way to estimate the curvature tensor of an induced connection directly from the original twistor data.

As an application, we consider a pair $X \hookrightarrow Y$ consisting of a complex contact 3-fold $(Y, L)$ and a Legendre submanifold $X=\mathrm{CP}^{1}$ such that the contact line bundle $L$ restricts to $X$ as $\mathcal{O}(3)$. Then the moduli space $M$ of all holomorphic Legendre deformations of $X$ inside $Y$ is a 4-dimensional manifold which comes equipped with an induced 1-flat $G_{3}$-structure $[\mathrm{Br} 2]$, and any such structure arises locally in this way. Suppose that there exists a line bundle $E \rightarrow Y$ such that $L=E^{\otimes 3}$ (such an $E$ always exists on a sufficiently small tubular neighbourhood of $X$ in $Y$ ). Since the normal bundle of $X$ is

Received June 4, 1997. 
isomorphic to $J^{1} \mathcal{O}(3)=\mathrm{C}^{2} \otimes \mathcal{O}(2)$, the above cohomology conditions on $\left.E\right|_{X_{t}} \otimes N_{t}^{*}$ are satisfied. Then the induced rank 2 vector bundle $\mathcal{E}$ comes equipped with a distinguished linear connection which, as easily follows from its integrability properties, is a "spinor" version of the torsion-free connection on $M$ with holonomy in $G_{3}$. In fact, any torsion-free connection with holonomy in $G_{3}$ can be constructed, at least locally, in this way (cf. [Me1]). The point is that the way we prove the above mentioned theoremconstruction gives a rather simple method of computing the distinguished linear connections from the twistor data $X \hookrightarrow Y$; in particular, one may use it to compute (as many as one would like!) explicit torsion-free affine connections with exotic holonomy $G_{3}$ (exotic in the sense that they are missing in the corresponding Berger list $[\mathrm{B}]$ ). Blowing up a point in the 2-fold covering of a neighbourhood of a $(1,4)$ curve $[\mathrm{Ped}]$ in a quadric $C \mathrm{P}^{1} \times C \mathrm{P}^{1}$, branched along the curve, we obtain a complex 2 -fold $Z$ containing a rational curve $X$ with self-intersection number 3 . Applying the developed machinery to the contact manifold $Y=\mathrm{P}\left(\Omega^{1} Z\right)$, we compute an explicit example of a torsionfree affine connection with holonomy $G_{3}$.

This example is in the class of deformation data " $X \hookrightarrow Y$ " where one should expect the most interesting applications of our theorem-contruction. The fact that a torsion-free affine connection with holonomy $G_{3}$ can be constructed by twistor methods is a particular manifestation of the general phenomenon [Me1]. Let $X$ be a generalised flag variety embedded as a Legendre submanifold into a complex contact manifold $Y$ with contact line bundle $L$ such that $L_{X}$ is very ample on $X$. Then the Legendre moduli space $M$ of the associated complete family $\left\{X_{t} \hookrightarrow Y \mid t \in M\right\}$ of all Legendre deformations of $X$ inside $Y$ is an $h^{0}\left(X, L_{X}\right)$-dimensional complex manifold [Me2]. Such a moduli space $M$ comes equipped with an induced irreducible $G$-structure with $G$ isomorphic to the connected component of the identity of the group of all global biholomorphisms $\phi: L_{X} \rightarrow L_{X}$ which commute with the projection $\pi: L_{X} \rightarrow X$. For motives explained in [Me1], irreducible $G$ structures which, at least locally, arise in this way are called Poisson. The class of Poisson $G$-structures is of interest for several reasons: (i) this class includes the subclass of all irreducible $G$-structures admitting torsion-free connections; (ii) the complement of the subclass in (i) consists of $G$-structures with rather special invariant torsion; (iii) the theorem of Hano-Ozeki $[\mathrm{H}-\mathrm{O}]$ is not true in the category of Poisson $G$-structures, i.e. the class of groups which can be realised as irreducibly acting holonomies of affine connections in Poisson $G$-structures is restricted. The main motivation behind our investigation of vector bundles on twistor spaces is that the resulting machinery gives an very efficient tool for the analysis of Poisson $G$-structures. The application to $G_{3}$-structures shows that, in some aspects, this 
machinery is much more effective than the Cartan-Kähler method used by Bryant [Br2] or the twistor method used in [Me1]. It is hoped that one might construct spinor versions of affine connections in Poisson $G$-structures by decomposing the contact line bundle $L_{X}=\left.\left.I_{1}^{\otimes m_{1}}\right|_{X} \otimes \cdots \otimes I_{k}^{\otimes m_{k}}\right|_{X}$ as a tensor product of the generators of the Picard group on the generalised flag variety $X$ and applying the theorem-construction to an appropriately assembled line bundle $E=I_{1}^{\otimes n_{1}} \otimes \cdots \otimes I_{k}^{\otimes n_{k}}$ with $n_{i} \leq m_{i}$.

In this paper we follow the tradition of identifying vector bundles with the associated locally free sheaves of local sections.

\section{Vector bundles on twistor spaces.}

2.1. Families of compact complex submanifolds. Let $Y$ and $M$ be complex manifolds and let $\pi_{1}: Y \times M \longrightarrow Y$ and $\pi_{2}: Y \times M \longrightarrow M$ be the natural projections. An analytic family of compact submanifolds of the complex manifold $Y$ with the moduli space $M$ is a complex submanifold $F \hookrightarrow Y \times M$ such that the restriction of the projection $\pi_{2}$ on $F$ is a proper regular map (regularity means that the rank of the differential of $\left.\nu \equiv \pi_{2}\right|_{F}: F \longrightarrow M$ is equal at every point to $\operatorname{dim} M$ ). Thus the family $F$ has the structure of a double fibration

$$
Y \stackrel{\mu}{\longleftarrow} F \stackrel{\nu}{\longrightarrow} M
$$

where $\left.\mu \equiv \pi_{1}\right|_{F}$. Thus for any point $t$ in the moduli space $M$ there is an associated submanifold $X_{t}$ in $Y$ which is said to belong to the family. On the other hand, for any point $y \in Y^{\prime} \equiv \cup_{t \in M} X_{t}$, there is an associated subset $\nu \circ \mu^{-1}(y)$ in $M$. It is not difficult to show that such a subset is always an analytic subspace of $M$. We denote the set of its regular points in $\nu \circ \mu^{-1}(y)$ by $\alpha_{y}$ and call it an alpha subspace of $M$ (cf. [Pen]). The ambient manifold $Y$ is often called a twistor space in this context.

If $F \hookrightarrow Y \times M$ is an analytic family of compact submanifolds, then, for any $t \in M$, there is a natural linear map [K]

$$
k_{t}: T_{t} M \longrightarrow H^{0}\left(X_{t}, N_{t}\right)
$$

from the tangent space at $t$ to the vector space of global holomorphic sections of the normal bundle $N_{t}=\left.T Y\right|_{X_{t}} / T X_{t}$ to the submanifold $X_{t} \hookrightarrow Y$, which can be described as follows. First note that the normal bundle of the embedding $\nu^{-1}(t) \hookrightarrow F$ is trivial and thus there is a canonical map $\rho_{t}: T_{t} M \longrightarrow H^{0}\left(\nu^{-1}(t), N_{\nu^{-1}(t) \mid F}\right)$. Then a composition $d \mu \circ \rho_{t}$ gives the desired map $k_{t}$ for the differential of $\mu$ maps global sections of $N_{\nu^{-1}(t) \mid F}$ to global sections of $N_{X_{t} \mid Y}$. Here the symbol $N_{A \mid B}$ stands for the normal bundle of a complex submanifold $A \hookrightarrow B$. 
Let $F \hookrightarrow Y \times M$ be a family of compact complex submanifolds and $N_{F}$ denote the normal bundle of the submanifold $F$ in $Y \times M$. It is clear that, for any $t \in M,\left.N_{F}\right|_{\nu^{-1}(t)} \simeq N_{t}$. Define $Y^{\prime}=\mu(F)$, i.e. $Y^{\prime}=\cup_{t \in M} X_{t}$. Then $F$ is a submanifold of $Y^{\prime} \times M$. It is not hard to check that there is a commutative diagram with exact rows and columns

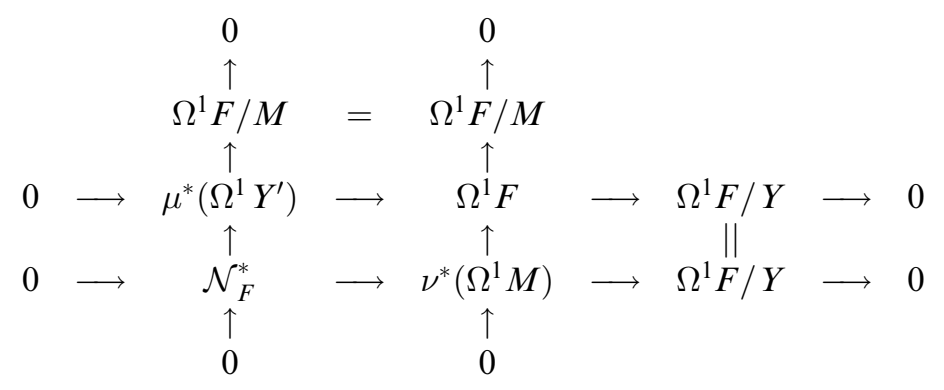

where $\mathcal{N}_{F}$ is the normal bundle of $F \hookrightarrow Y^{\prime} \times M$, and $\Omega^{1} F / Y$ and $\Omega^{1} F / M$ are vector bundles of $\mu$-vertical and, respectively, $\nu$-vertical 1 -forms. It is clear that, for any $t \in M, \Omega^{1} F /\left.M\right|_{\nu^{-1}(t)} \simeq \Omega^{1} X_{t}$ and $\left.\mathcal{N}_{F}\right|_{\nu^{-1}(t)} \simeq \mathcal{N}_{t}$, where $\mathcal{N}_{t}$ is the normal bundle of $X_{t}$ in $Y^{\prime}$. It is a subbundle of $N_{t}$ generated by global sections from $k\left(T_{t} M\right) \subset H^{0}\left(X_{t}, N_{t}\right)$. In particular, if the natural "evaluation" map

$$
k\left(T_{t} M\right) \otimes_{\mathrm{C}} \mathcal{O}_{X_{t}} \longrightarrow N_{t}
$$

is an epimorphism, then $\mathcal{N}_{t}=N_{t}$ and one obtains a commutative diagram as above with $Y^{\prime}$ and $\mathcal{N}_{F}$ replaced by $Y$ and $N_{F}$ respectively (cf. [B-E]). In all examples of families of compact complex submanifolds ever considered in the twistor theory framework, this condition holds.

We shall be interested in this paper in two kinds of maximal families of compact complex submanifolds. The first one was introduced by Kodaira in 1962. The initial data is a compact complex submanifold $X$ of a complex manifold $Y$ with normal bundle $N$ and the object of study is the associated set, $M$, of all "nearby" compact complex submanifolds, $X_{t} \hookrightarrow Y$, obtained by holomorphic deformations of $X$ inside $Y$. If $H^{1}(X, N)=0$, then, according to Kodaira [K], $X$ belongs to the maximal analytic family $F \hookrightarrow Y \times M$ with the moduli space $M$ being an $h^{0}(X, N)$-dimensional complex manifold. Moreover, here the canonical map $k_{t}: T_{t} M \longrightarrow H^{0}\left(X_{t}, N_{t}\right)$ is an isomorphism for any $t \in M$. The manifold $M$ is called a Kodaira moduli space. It is not hard to show that if the natural evaluation map

$$
H^{0}\left(X_{t}, N_{t}\right) \otimes \mathcal{O}_{X_{t}} \longrightarrow N_{t}
$$

is an epimorphism for all $t \in \nu \circ \mu^{-1}(y)$, then the subspace $\nu \circ \mu^{-1}(y) \subset M$ 
has no singularities, i.e. it coincides with the alpha subspace $\alpha_{y}$. Also, in this case $\mathcal{N}_{t}=N_{t}$.

The second type of maximal family of compact complex submanifolds which will be of interest to us arises from different initial data " $X \hookrightarrow Y$ ". Assume that the ambient manifold ("twistor space") $Y$ has a complex contact structure, i.e. a corank 1 holomorphic distribution $D \subset T Y$ such that the Frobenius form

$$
\begin{aligned}
\Phi: D \times D & \longrightarrow T Y / D \\
(v, w) & \longrightarrow[v, w] \bmod D
\end{aligned}
$$

is non-degenerate. Then $\operatorname{dim} Y=2 n+1$ for some integer $n \geq 1$ and there is a natural contact line bundle $L$ on $Y$ defined by the exact sequence

$$
0 \longrightarrow D \longrightarrow T Y \longrightarrow L \longrightarrow 0 .
$$

Assume also that the submanifold $X \hookrightarrow Y$ is a Legendre submanifold, i.e. $\operatorname{dim} X=n$ and $T X \subset D$. The normal bundle of a Legendre submanifold $X \hookrightarrow Y$ is isomorphic to $J^{1} L_{X}$, where $L_{X}=\left.L\right|_{X}$, and fits, therefore, into the following exact sequence

$$
0 \longrightarrow \Omega^{1} X \otimes L_{X} \longrightarrow N \stackrel{p r}{\longrightarrow} L_{X} \longrightarrow 0 .
$$

The object of study is the set $M$ of all "nearby" complex Legendre submanifolds of $Y$ obtained by holomorphic deformations of $X$ inside $Y$. It has been proved in [Me1,Me2] that if $H^{1}\left(X, L_{X}\right)=0$, then $X$ belongs to a maximal family, $F \subset Y \times M$, of compact complex Legendre submanifolds with the moduli space $M$ being an $h^{0}\left(X, L_{X}\right)$-dimensional complex manifold. Moreover, the canonical composition

$$
T_{t} M \stackrel{k_{t}}{\longrightarrow} H^{0}\left(X_{t}, N_{t}\right) \stackrel{p r}{\longrightarrow} H^{0}\left(X_{t}, L_{X_{t}}\right)
$$

is an isomorphism for any $t \in M$. The manifold $M$ is called a Legendre moduli space. Thus each point $t$ in $M$ is represented by a compact complex Legendre submanifold $X_{t}$ in $Y$, while each point $y$ in $Y^{\prime} \equiv \cup_{t \in M} X_{t}$ is represented by an alpha subspace $\alpha_{y}$ in $M$. It is not hard to show that if the natural "derivation and then evaluation" map

$$
H^{0}\left(X_{t}, L_{X_{t}}\right) \otimes \mathcal{O}_{X_{t}} \longrightarrow J^{1} L_{X_{t}}
$$

is an epimorphism for all $t$, then for any $y \in Y^{\prime}$ the alpha subspace $\alpha_{y}$ coincides with $\nu \circ \mu^{-1}(y)$, the set of all Legendre submanifolds passing through $y$. Also, in this case $\mathcal{N}_{t}=N_{t}$.

If $X \hookrightarrow Y$ is a complex submanifold, there is an exact sequence of vector bundles

$$
\left.0 \longrightarrow N^{*} \longrightarrow \Omega^{1} Y\right|_{X} \longrightarrow \Omega^{1} X \longrightarrow 0
$$


which induces a natural embedding $\mathrm{P}\left(N^{*}\right) \hookrightarrow \mathrm{P}\left(\Omega^{1} Y\right)$ of total spaces of the associated projectivised bundles. The manifold $\hat{Y}=\mathrm{P}\left(\Omega^{1} Y\right)$ carries a natural contact structure such that the constructed embedding $\hat{X}=\mathrm{P}\left(N^{*}\right) \hookrightarrow \hat{Y}$ is a Legendre one [Ar]. It is not hard to show [Me1,Me2] that if $\left\{X_{t} \hookrightarrow Y \mid t \in M\right\}$ is a maximal Kodaira family of compact submanifolds, then the associated family $\left\{\hat{X}_{t}=\mathrm{P}\left(N_{t}^{*}\right) \hookrightarrow \mathrm{P}\left(\Omega^{1} Y\right) \mid t \in M\right\}$ of projectivized conormal bundles is a maximal family of compact Legendre submanifolds.

2.2. Geometric structures induced from vector bundles on twistor spaces. Let $F \hookrightarrow Y \times M$ be a family of compact complex submanifolds. A holomorphic vector bundle $E$ on $Y$ is said to be weakly $M$-uniform ${ }^{1}$ if the function

$$
\begin{aligned}
M & \longrightarrow \\
t & \longrightarrow h^{0}\left(X_{t},\left.E\right|_{X_{t}}\right)
\end{aligned}
$$

is globally constant on $M$. Then using the double fibration

$$
Y \stackrel{\mu}{\longleftarrow} F \stackrel{\nu}{\longrightarrow} M,
$$

one may associate to $E \rightarrow Y$ a locally free sheaf $\mathcal{E}=\nu_{*}^{0}\left(\mu^{*}(E)\right)$ on $M$. It is natural to call $\mathcal{E}$ induced from the weakly $M$-uniform bundle $E$. For any $t \in M$, the corresponding fibre $\mathcal{E}_{t} \subset \mathcal{E}$ is isomorphic to $H^{0}\left(X_{t},\left.E\right|_{X_{t}}\right)$. If $E$ is non-trivial on $X_{t}$, then, for any $y \in Y^{\prime}$, the restriction $\left.\mathcal{E}\right|_{\alpha_{\nu}}$ of $\mathcal{E}$ to the alpha surface $\alpha_{y}$ has canonically a vector subbundle $\mathfrak{T}_{y}$ whose fibre at any $t \in \alpha_{y}$ is, by definition, the subspace of $H^{0}\left(X_{t},\left.E\right|_{X_{t}}\right)$ consisting of global sections which vanish at $y \in Y^{\prime}$. We also define $\mathfrak{S}_{y}=\left.\mathcal{E}\right|_{\alpha_{y}} / \mathfrak{T}_{y}$. The following result is a variation on the themes developed in [W,M,E,B-E,Me3].

THEOREM 1. Let $F \hookrightarrow Y \times M$ be a family of compact complex submanifolds and $E$ a weakly $M$-uniform vector bundle on $Y$. If

$$
H^{0}\left(X_{t},\left.E\right|_{X_{t}} \otimes \mathcal{N}_{t}^{*}\right)=H^{1}\left(X_{t},\left.E\right|_{X_{t}} \otimes \mathcal{N}_{t}^{*}\right)=0
$$

for all $t \in M$, then the induced vector bundle $\mathfrak{E}$ on $M$ comes equipped canonically with an induced linear connection $\nabla$ such that

(i) At each $t \in M$, the curvature tensor of $\nabla$ is represented by an element of $H^{1}\left(X_{t},\left.E\right|_{X_{t}} \otimes \odot^{2} \mathcal{N}_{t}^{*}\right) \otimes\left(H^{0}\left(X_{t},\left.E\right|_{X_{t}}\right)\right)^{*}$;

(ii) For any $y \in Y^{\prime}$, the induced connection $\left.\nabla\right|_{\alpha_{y}}$ preserves the vector subbundle $\left.\mathfrak{T}_{y} \subset \mathfrak{E}\right|_{\alpha_{y}}$, i.e. the following covariant differentials

$$
\nabla_{y}^{\prime}: \mathfrak{T}_{y} \rightarrow \mathfrak{T}_{y} \otimes \Omega^{1} \alpha_{y} \quad \nabla_{y}^{\prime \prime}: \mathfrak{S}_{y} \rightarrow \mathfrak{S}_{y} \otimes \Omega^{1} \alpha_{y}
$$

\footnotetext{
${ }^{1}$ If $F$ is a family of compact rigid manifolds (say, of generalized flag varieties), then one might consider an $M$-uniform vector bundle $E$ on $Y$ whose restrictions $\left.E\right|_{X}$ have, by definition, the same holomorphy type for all $t \in M$ [E, B-E].
} 
are well defined;

(iii) For any $y \in Y^{\prime}$, the induced connection $\nabla_{y}^{\prime \prime}$ is flat.

Proof. Consider the composition

$$
d_{F / Y}: \mathcal{O}_{F} \stackrel{d}{\longrightarrow} \Omega^{1} F \longrightarrow \Omega^{1} F / Y
$$

and notice that it extends naturally to a well-defined differential operator

$$
\nabla_{F / Y}: \mu^{*}(E) \longrightarrow \mu^{*}(E) \otimes \Omega^{1} F / Y
$$

which annuls the subsheaf $\mu^{-1}(E) \subset \mu^{*}(E)$. The diagram (1) implies the following exact sequence

$$
0 \longrightarrow \mu^{*}(E) \otimes \mathcal{N}_{F}^{*} \longrightarrow \mu^{*}(E) \otimes \nu^{*}\left(\Omega^{1} M\right) \longrightarrow \mu^{*}(E) \otimes \Omega^{1} F / Y \longrightarrow 0
$$

whose associated long exact sequence of direct image sheaves contains the following piece

$\ldots \longrightarrow \nu_{*}^{0}\left(\mu^{*}(E) \otimes \mathcal{N}_{F}^{*}\right) \longrightarrow \mathcal{E} \otimes \Omega^{1} M \longrightarrow \nu_{*}^{0}\left(\mu^{*}(E) \otimes \Omega^{1} F / Y\right) \longrightarrow \nu_{*}^{1}\left(\mu^{*}(E) \otimes \mathcal{N}_{F}^{*}\right) \longrightarrow \ldots$

Both $\nu_{*}^{0}\left(\mu^{*}(E) \otimes \mathcal{N}_{F}^{*}\right)$ and $\nu_{*}^{1}\left(\mu^{*}(E) \otimes \mathcal{N}_{F}^{*}\right)$ are locally free on $M$ with the fibre at $t \in M$ isomorphic, respectively, to $H^{0}\left(X_{t},\left.E\right|_{X_{t}} \otimes \mathcal{N}_{t}^{*}\right)$ and $H^{1}\left(X_{t}\right.$, $\left.\left.E\right|_{X_{t}} \otimes \mathcal{N}_{t}^{*}\right)$. Since the latter two cohomology groups vanish by assumption, we get a canonical isomorphism

$$
\nu_{*}^{0}\left(\mu^{*}(E) \otimes \Omega^{1} F / Y\right)=\mathcal{E} \otimes \Omega^{1} M .
$$

Then the descent of the diagram $\nabla_{F / Y}: \mu^{*}(E) \longrightarrow \mu^{*}(E) \otimes \Omega^{1} F / Y$ from $F$ to $M$ induces a first order covariant differential

$$
\nabla=\mu_{*}\left(\nabla_{F / Y}\right): \mathcal{E} \longrightarrow \mathcal{E} \otimes \Omega^{1} M .
$$

Thus the fact that $\mathcal{E}$ comes equipped with an induced linear connection $\nabla$ is almost obvious. In order to prove that $\nabla$ has properties stated in items (i)(iii) above, we shall discuss next a local coordinate version of the above "diagramatic" construction of $\nabla$. The coordinate approach, though apparently more dull, provides us not only with a rather elementary proof of Theorem 1, but also with a more suitable reformulation (see Proposition 4 below) as well as with the inverse construction (see subsection 2.4). This approach is also useful in the context of the explicit construction of $\nabla$ in examples of interest (see section 4).

We may assume without loss of generality that the moduli space $M$ is a ball in $\mathrm{C}^{m}$ with coordinates $\left\{t^{\alpha}\right\}$ (the obvious functoriality of the construction of $\nabla$ given below makes it clear how to deal with the general case). Fix any $t_{0} \in M$ and consider the associated submanifold $X_{0}=\mu \circ \nu^{-1}(t) \hookrightarrow Y$. There is a finite Leray covering $\left\{W_{\mathrm{i}}\right\}$ of a sufficiently small open neighbourhood of 
$X_{0}$ in $Y$ by coordinate charts $\left\{W_{\mathrm{i}} \mid \mathrm{i} \in I\right\}$ with local coordinate functions ${ }^{2}$, $\left(w_{\mathrm{i}}^{a}, z_{\mathrm{i}}^{m}\right)$, on each chart $W_{\mathrm{i}}$ such that the intersection $X_{0} \cap W_{\mathrm{i}}$ is given by

$$
w_{\mathrm{i}}^{a}=0 .
$$

On the intersection $W_{\mathrm{i}} \cap W_{\mathrm{j}}$ the coordinates $w_{\mathrm{i}}^{a}, z_{\mathrm{i}}^{m}$ are holomorphic functions of $w_{\mathrm{j}}^{b}$ and $z_{\mathrm{j}}^{n}$,

$$
w_{\mathrm{i}}^{a}=f_{\mathrm{ij}}^{a}\left(w_{\mathrm{j}}^{b}, z_{\mathrm{j}}^{n}\right), \quad z_{\mathrm{i}}^{m}=g_{\mathrm{ij}}^{m}\left(w_{\mathrm{j}}^{b}, z_{\mathrm{j}}^{n}\right),
$$

with $f_{\mathrm{ij}}^{a}\left(0, z_{\mathrm{j}}^{n}\right)=0$.

The collection of coordinate domains $V_{\mathrm{i}}=W_{\mathrm{i}} \times M$ with coordinate functions $\left(w_{\mathrm{i}}^{a}, z_{\mathrm{i}}^{m}, t^{\alpha}\right)$ is a Leray covering of an open neighbourhood of $X_{0} \times M$ in $Y \times M$. Shrinking the coordinate ball $M \subset \mathrm{C}^{\operatorname{dim} M}$ as necessary and using the implicit function theorem, we may describe the submanifold $F \hookrightarrow Y \times M$ in each coordinate chart $V_{\mathrm{i}}$ by simultaneous equations of the form ([K])

$$
w_{\mathrm{i}}^{a}=\phi_{\mathrm{i}}^{a}\left(z_{\mathrm{i}}, t\right),
$$

where $\phi_{\mathrm{i}}^{a}\left(z_{\mathrm{i}}, t\right)$ are holomorphic functions of $z_{\mathrm{i}}=\left(z_{\mathrm{i}}^{m}\right)$ and $t=\left(t^{\alpha}\right)$ which satisfy the conditions $\phi_{\mathrm{i}}^{a}\left(z_{\mathrm{i}}, t_{0}\right)=0$. For each fixed $t \in M$ the equation $w_{\mathrm{i}}^{a}=\phi_{\mathrm{i}}^{a}\left(z_{\mathrm{i}}, t\right)$ defines a submanifold $X_{t} \cap W_{\mathrm{i}} \hookrightarrow W_{\mathrm{i}}$.

Thus we have constructed a finite Leray covering of $F$ by coordinate neighbourhoods $\left\{V_{\mathrm{i}}\right\}$ with local coordinate functions $\left(z_{\mathrm{i}}^{a}, t^{\alpha}\right)$ which are related to each other on the overlaps $V_{\mathrm{i}} \cap V_{\mathrm{j}}$ as follows

$$
z_{\mathrm{i}}^{m}=g_{\mathrm{ij}}^{m}\left(\phi_{\mathrm{j}}\left(z_{\mathrm{j}}, t\right), z_{\mathrm{j}}\right),
$$

where $\phi_{\mathrm{j}}\left(z_{\mathrm{j}}, t\right)=\left(\phi_{\mathrm{j}}^{a}\left(z_{\mathrm{j}}, t\right)\right)$. Obviously we have

$$
\phi_{\mathrm{i}}^{a}\left(g_{\mathrm{ij}}\left(\phi_{\mathrm{j}}\left(z_{\mathrm{j}}, t\right), z_{\mathrm{j}}\right), t\right)=f_{\mathrm{ij}}^{a}\left(\phi_{\mathrm{j}}\left(z_{\mathrm{j}}, t\right), z_{\mathrm{j}}\right) .
$$

Define the functions

$$
w_{t \mathrm{i}}^{a}=w_{\mathrm{i}}^{a}-\phi_{\mathrm{i}}^{a}\left(z_{\mathrm{i}}, t\right)
$$

and note that the collections of functions $\left(w_{t \mathrm{i}}^{a}, z_{\mathrm{i}}^{m}, t^{\alpha}\right)$ form a local coordinate system on $Y \times M$ with the property that the submanifold $F \hookrightarrow Y \times M$ is described in each chart $V_{\mathrm{i}}$ by the equations $w_{t \mathrm{i}}^{a}=0$. On the intersection $V_{\mathrm{i}} \cap V_{\mathrm{j}}$ we have

\footnotetext{
${ }^{2}$ Here and throughout the paper small Latin indices from the first half of the alphabet, $a, b, c \ldots$, take values $1, \ldots \operatorname{codim} X_{t}$; small Latin indices from the second half of the alphabet, $l, m, n, \ldots$, take values $1, \ldots \operatorname{dim} X_{t}$; capital Latin indices, $A, B, C \ldots$, take values $1, \ldots, \operatorname{rank\mathcal {E}}$; calligraphic indices $\mathcal{A}, \mathcal{B}, \mathcal{C}, \ldots$, take values $1, \ldots, \operatorname{rank} E$; and Greek indices take values $1, \ldots$, $\operatorname{dim} M$.
} 


$$
\begin{aligned}
w_{t \mathrm{i}}^{a} & =f_{\mathrm{ij}}^{a}\left(w_{t \mathrm{j}}+\phi_{\mathrm{j}}\left(z_{\mathrm{j}}, t\right), z_{\mathrm{j}}\right)-\phi_{\mathrm{i}}^{a}\left(g_{\mathrm{ij}}\left(w_{t \mathrm{j}}+\phi_{\mathrm{j}}\left(z_{\mathrm{j}}, t\right), z_{\mathrm{j}}\right), t\right), \\
z_{\mathrm{i}}^{m} & =g_{\mathrm{ij}}^{m}\left(w_{t \mathrm{j}}+\phi_{\mathrm{j}}\left(z_{\mathrm{j}}, t\right), z_{\mathrm{j}}\right) .
\end{aligned}
$$

The normal bundle $N_{F}$ is isomorphic to $\left(J_{F} / J_{F}^{2}\right)^{*}$, where $J_{F}$ is the ideal sheaf of functions on $Y \times M$ which vanish on $F$. Since $J_{F}$ is generated over $V_{\mathrm{i}}$ by $w_{t \mathrm{i}}^{a}$, a general element $\sigma$ in $H^{0}\left(F, N_{F}\right)$ can be represented by a Čech 0 cocycle, $\left\{\sigma_{\mathrm{i}}^{a}\left(z_{\mathrm{i}}, t\right)\right\}$, of vector-valued holomorphic functions which are "glued" on $V_{\mathrm{i}} \cap V_{\mathrm{j}}$ according to the rule

$$
\sigma_{\mathrm{i}}^{a}=F_{\mathrm{ij} b}^{a} \sigma_{\mathrm{j}}^{b},
$$

where the functions,

$$
\left.F_{\mathrm{ij} b}^{a} \equiv \frac{\partial w_{t \mathrm{i}}^{a}}{\partial w_{t \mathrm{j}}^{b}}\right|_{w_{t \mathrm{j}}=0}=\left.\frac{\partial f_{\mathrm{ij}}^{a}}{\partial w_{\mathrm{j}}^{b}}\right|_{w_{\mathrm{j}}=\phi\left(z_{\mathrm{j}}, t\right)}-\left.\left.\frac{\partial \phi_{\mathrm{i}}^{a}}{\partial z_{\mathrm{i}}^{m}}\right|_{z_{\mathrm{i}}=g_{\mathrm{ij}}\left(\phi_{\mathrm{j}}, z_{\mathrm{j}}\right)} \frac{\partial g_{\mathrm{ij}}^{m}}{\partial w_{\mathrm{j}}^{b}}\right|_{w_{\mathrm{j}}=\phi_{\mathrm{j}}\left(z_{\mathrm{j}}, t\right)},
$$

form a Čech 1-cocycle, $F_{\mathrm{ik} b}^{a}=F_{\mathrm{ij} c}^{a} F_{\mathrm{jk} b}^{c}$, representing the isomorphism class of $N_{F}$ as a cohomology class in $H^{1}\left(F, G L\left(\operatorname{codim} X, \mathcal{O}_{F}\right)\right)$. The Kodaira isomorphism

$$
k: T M \longrightarrow \nu_{*}^{0}\left(N_{F}\right)
$$

can now be described very explicitly: take any vector field $v$ on $M$ and apply the associated first-order differential operator $V^{\alpha} \partial_{\alpha}$ to each function $\phi_{\mathrm{i}}^{a}\left(z_{\mathrm{i}}, t\right)$, where $\partial_{\alpha}=\partial / \partial t^{\alpha}$. The result is a 0 -cochain $\left\{\sigma_{\mathrm{i}}^{a}=V^{\alpha} \partial_{\alpha} \phi_{\mathrm{i}}^{a}\left(z_{\mathrm{i}}, t\right)\right\}$ which, as it is not hard to show, satisfies the cocycle condition (3) thus representing an element $k(v) \in H^{0}\left(F, N_{F}\right)$.

Without loss of generality we may assume that the bundle $E$ can be trivialised over each $W_{\mathrm{i}}$, with fibre coordinates $s_{\mathrm{i}}^{\mathcal{A}}, \mathcal{A}=1, \ldots, \operatorname{rank} E$. Then, on the intersections $W_{\mathrm{i}} \cap W_{\mathrm{j}}$,

$$
s_{\mathrm{i}}^{\mathcal{A}}=G_{\mathrm{ij} \mathcal{B}}^{\mathcal{A}} s_{\mathrm{j}}^{\mathcal{B}},
$$

where $\left\{G_{\mathrm{ij}} \mathcal{\mathcal { A }}\left(w_{\mathrm{j}}, z_{\mathrm{j}}\right)\right\}$ is a C̆ech 1-cocycle representing, in the Leray covering $\left\{W_{\mathrm{i}}\right\}$ of $Y$, the cohomology class in $H^{1}\left(Y, G L\left(\operatorname{rank} E, \mathcal{O}_{Y}\right)\right)$ associated to the isomorphism class of $E$. The sheaf $\mathcal{E}=\nu_{*}^{0}\left(\mu^{*}(E)\right)$ is a free $\mathcal{O}_{M}$-module on $F$. Let $\left\{e_{A}\right\}, A=1, \ldots, \operatorname{rank} \mathcal{E}$, be a set of basis sections of $\mathcal{E}$ over $M$. Each section $e_{A} \in H^{0}(M, \mathcal{E}) \simeq H^{0}\left(F, \mu^{*}(E)\right)$ can be represented, in the covering $\left\{V_{\mathrm{i}}\right\}$ of $F$, by a Čech 0 -cochain $\left\{\lambda_{\mathrm{i} A}^{\mathcal{A}}\right\}$ with coefficients in $\mu^{*}(E)$ satisfying the cocycle condition

$$
\lambda_{\mathrm{i} A}^{\mathcal{A}}\left(z_{\mathrm{i}}, t\right)=\left.G_{\mathrm{ij} \mathcal{B}}^{\mathcal{A}}\left(w_{\mathrm{j}}, z_{\mathrm{j}}\right)\right|_{w_{\mathrm{j}}=\phi_{\mathrm{j}}\left(z_{\mathrm{j}}, t\right)} \lambda_{\mathrm{j} A}^{\mathcal{B}}\left(z_{\mathrm{j}}, t\right) .
$$

Taking the partial derivative of this equation with respect to $t^{\alpha}$, one obtains 


$$
\frac{\partial \lambda_{\mathrm{i} A}^{\mathcal{A}}}{\partial t^{\alpha}}=\left.G_{\mathrm{ij} \mathcal{B}}^{\mathcal{A}}\right|_{w_{\mathrm{j}}=\phi_{\mathrm{j}}} \frac{\partial \lambda_{\mathrm{j} A}^{\mathcal{B}}}{\partial t^{\alpha}}+\left[\left.\frac{\partial G_{\mathrm{ij}}^{\mathcal{A} \mathcal{B}}}{\partial w_{\mathrm{j}}^{b}}\right|_{w_{\mathrm{j}}=\phi_{\mathrm{j}}} \lambda_{\mathrm{j} A}^{\mathcal{B}}-\left.\frac{\partial \lambda_{\mathrm{i} A}^{\mathcal{A} A}}{\partial z_{\mathrm{i}}^{m}} \frac{\partial g_{\mathrm{ij}}^{m}}{\partial w_{\mathrm{j}}^{b}}\right|_{w_{\mathrm{j}}=\phi_{\mathrm{j}}}\right] \frac{\partial \phi_{\mathrm{j}}^{b}}{\partial t^{\alpha}}
$$

$\left(z_{\mathrm{j}}\right.$ being held constant). Denote

$$
\chi_{\mathrm{ij} b A}^{\mathcal{A}}=\left.\frac{\partial G_{\mathrm{ij}}^{\mathcal{A}}}{\partial w_{\mathrm{j}}^{b}}\right|_{w_{\mathrm{j}}=\phi_{\mathrm{j}}} \lambda_{\mathrm{j} A}^{\mathcal{B}}-\left.\frac{\partial \lambda_{\mathrm{i}}^{\mathcal{A} A}}{\partial z_{\mathrm{i}}^{m}} \frac{\partial g_{\mathrm{ij}}^{m}}{\partial w_{\mathrm{j}}^{b}}\right|_{w_{\mathrm{j}}=\phi_{\mathrm{j}}} .
$$

There is a natural projection

$$
\mu^{*}(E) \otimes N_{F}^{*} \stackrel{i}{\longrightarrow} \mu^{*}(E) \otimes \mathcal{N}_{F}^{*} \longrightarrow 0 .
$$

Since in equation (5) the functions $\chi_{\mathrm{ij} b A}^{\mathcal{A}}$ are contracted with $\partial \phi_{\mathrm{j}} / \partial t^{\alpha}$, it is their images under the projection $i$ that are of interest to us.

Lemma 2. The 1-cochain $\left\{i\left(\chi_{\mathrm{ij} b A}^{\mathcal{A}}\right)\right\}$ with coefficients in $\mu^{*}(E) \otimes \mathcal{N}_{F}^{*} \otimes \nu^{*}\left(\mathcal{E}^{*}\right)$ is a 1-cocycle.

Proof. Suppose

$$
\delta\left\{\chi_{\mathrm{ij} b A}^{\mathcal{A}}\right\}=\left\{\psi_{\mathrm{ijk} b A}^{\mathcal{A}}\right\}
$$

for some 2-cochain of functions $\psi_{\mathrm{ijk} b A} \underset{\mathcal{A}}{ }$ with coefficients in $\mu^{*}(E) \otimes N_{F}^{*} \otimes$ $\nu^{*}\left(\mathcal{E}^{*}\right)$. Then from equations (5) it follows that

$$
\psi_{\mathrm{ijk} k A} \mathcal{A} \frac{\partial \phi_{\mathrm{j}}^{b}}{\partial t^{\alpha}}=0 .
$$

Since the map $\nu^{*}(T M) \longrightarrow \mathcal{N}_{F}$ is an epimorphism, the latter equation implies

$$
i\left(\psi_{\mathrm{ijk} b A}^{\mathcal{A}}\right)=0
$$

which in turn implies that the 1-cochain $\left\{i\left(\chi_{\mathrm{ij} b A}^{\mathcal{A}}\right)\right\}$ is a 1-cocycle representing thus a cohomology class in $H^{1}\left(F, \mu^{*}(E) \otimes \mathcal{N}_{F}^{*} \otimes \nu^{*}\left(\mathcal{E}^{*}\right)\right)$.

Since, by assumption, $H^{0}\left(X_{t},\left.E\right|_{X_{t}} \otimes \mathcal{N}_{t}^{*}\right)=H^{1}\left(X_{t},\left.E\right|_{X_{t}} \otimes \mathcal{N}_{t}^{*}\right)=0$ for all $t \in M$, the Leray spectral sequence for $\nu$ implies

$$
H^{0}\left(\mu^{*}(E) \otimes \mathcal{N}_{F}^{*} \otimes \nu^{*}\left(\mathcal{E}^{*}\right)\right)=H^{1}\left(\mu^{*}(E) \otimes \mathcal{N}_{F}^{*} \otimes \nu^{*}\left(\mathcal{E}^{*}\right)\right)=0 .
$$

Therefore, the Čech 1-cocycle $\left\{i\left(\chi_{\mathrm{ij} b A}^{\mathcal{A}}\right)\right\}$ is a coboundary of a uniquely determined 0-cochain $\left\{\theta_{\mathrm{i} b A}^{\mathcal{A}}\right\}$, i.e.

$$
i\left(\chi_{\mathrm{ij} b A}^{\mathcal{A}}\right)=-\theta_{\mathrm{i} c A}^{\mathcal{A}} F_{\mathrm{ij} b}^{c}+G_{\mathrm{ij} \mathcal{B}}^{\mathcal{A}} \theta_{\mathrm{j} b A}^{\mathcal{B}}
$$

Then equation (5) can be rewritten in the form

$$
\frac{\partial \lambda_{\mathrm{i} A}^{\mathcal{A}}}{\partial t^{\alpha}}+\theta_{\mathrm{i} b A}^{\mathcal{A} A} \frac{\partial \phi_{\mathrm{i}}^{b}}{\partial t^{\alpha}}=\left.G_{\mathrm{ij} \mathcal{B}}^{\mathcal{A}}\right|_{w_{\mathrm{j}}=\phi_{\mathrm{j}}}\left(\frac{\partial \lambda_{\mathrm{j} A}^{\mathcal{B}}}{\partial t^{\alpha}}+\theta_{\mathrm{j} b A}^{\mathcal{B} A} \frac{\partial \phi_{\mathrm{j}}^{b}}{\partial t^{\alpha}}\right)
$$


which implies that the Čech cochain

$$
\left\{\frac{\partial \lambda_{\mathrm{i} A}^{\mathcal{A}}}{\partial t^{\alpha}}+\theta_{\mathrm{i} b A} \mathcal{A} \frac{\partial \phi_{\mathrm{i}}^{b}}{\partial t^{\alpha}}\right\}
$$

is a 0 -cocycle representing, for each $A$ and $\alpha$, a global section of $\mu^{*}(E)$ over $F$, i.e. a section of $\mathcal{E}$ over $M$. Then

$$
\frac{\partial \lambda_{\mathrm{i} A}^{\mathcal{A}}\left(z_{\mathrm{i}}, t\right)}{\partial t^{\alpha}}+\theta_{\mathrm{i} b A}^{\mathcal{A}}\left(z_{\mathrm{i}}, t\right) \frac{\partial \phi_{\mathrm{i}}^{b}\left(z_{\mathrm{i}}, t\right)}{\partial t^{\alpha}}=\Gamma_{\alpha A}^{B}(t) \lambda_{\mathrm{i} B}^{\mathcal{A}}\left(z_{\mathrm{i}}, t\right)
$$

for some holomorphic functions $\Gamma_{\alpha A}^{B}(t)$ on $M$. It is clear that the latter are nothing but the coordinate coefficients of the induced connection $\nabla$. Taking the partial derivative of this equation with respect to $t^{\beta}$ and then antisymmetrising on $\alpha$ and $\beta$ one obtains the equality

$$
\nabla_{\alpha} \theta_{\mathrm{i} b A}^{\mathcal{A} A} \frac{\partial \phi_{\mathrm{j}}^{b}}{\partial t^{\beta}}-\nabla_{\beta} \theta_{\mathrm{i} b A}^{\mathcal{A} A} \frac{\partial \phi_{\mathrm{j}}^{b}}{\partial t^{\alpha}}=F_{\alpha \beta A}^{B} \lambda_{\mathrm{i} B}^{\mathcal{A}}
$$

where

$$
\nabla_{\alpha} \theta_{\mathrm{i} b A}^{\mathcal{A}}=\partial_{\alpha} \theta_{\mathrm{i} b A}^{\mathcal{A}}-\Gamma_{\alpha A}^{B} \theta_{\mathrm{i} b B}^{\mathcal{A}}
$$

and

$$
F_{\alpha \beta A}^{B}=\partial_{\alpha} \Gamma_{\beta A}^{B}-\partial_{\beta} \Gamma_{\alpha A}^{B}+\Gamma_{\alpha A}^{C} \Gamma_{\beta C}^{B}-\Gamma_{\beta A}^{C} \Gamma_{\alpha C}^{B}
$$

is the curvature tensor of the induced connection. Straightforward calculations show that

$$
\delta\left\{\nabla_{\alpha} \theta_{\mathrm{i} a A}^{\mathcal{A}}\right\}=\left\{\rho_{\mathrm{ij} a b A}^{\mathcal{A}} \frac{\partial \phi_{\mathrm{j}}^{b}}{\partial t^{\alpha}}\right\}
$$

for some 1-cochain $\left\{\rho_{\mathrm{ij} a b A}^{\mathcal{A}}\right\}$ on $F$ with coefficients in $\mu^{*}(E) \otimes \odot^{2} N_{F}^{*} \otimes \nu^{*}\left(\mathcal{E}^{*}\right)$. Then the statement (i) of the Theorem follows immediately from the invariance of equation (8) under transformations

$$
\nabla_{\alpha} \theta_{\mathrm{i} a A}^{\mathcal{A}} \longrightarrow \nabla_{\alpha} \theta_{\mathrm{i} a A}^{\mathcal{A}}+\varepsilon_{\mathrm{i} a b A}^{\mathcal{A} A} \frac{\partial \phi_{\mathrm{j}}^{b}}{\partial t^{\alpha}}
$$

for any $\underset{\mathrm{i} a b A}{\mathcal{A} A} \in \Gamma\left(V_{\mathrm{i}}, \mu^{*}(E) \otimes \odot^{2} N_{F}^{*} \otimes \nu^{*}\left(\mathcal{E}^{*}\right)\right)$ and the following

Lemma 3. The 1-cochain $\left\{i\left(\rho_{\mathrm{ij} a b A}^{\mathcal{A}}\right)\right\}$ with coefficients in $\mu^{*}(E) \otimes \odot^{2} \mathcal{N}_{F}^{*} \otimes$ $\nu^{*}\left(\mathcal{E}^{*}\right)$ is a 1-cocycle.

Proof. Suppose

$$
\delta\{\underset{\mathrm{ij} a b A}{\stackrel{\mathcal{A}}{ }}\}=\left\{\underset{\mathrm{ijk} a b A}{\left.\stackrel{\mathcal{A}}{{ }^{2}}\right\}}\right\}
$$


for some 2-cochain of functions $\sigma_{\mathrm{ijk} a b A}^{\mathcal{A}}$ with coefficients in $\mu^{*}(E) \otimes \odot^{2} N_{F}^{*} \otimes$ $\nu^{*}\left(\mathcal{E}^{*}\right)$. Then from equations (9) it follows that

$$
\underset{\mathrm{ijk} a b A}{\mathcal{A} A} \frac{\partial \phi_{\mathrm{j}}^{b}}{\partial t^{\alpha}}=0
$$

which implies that

$$
i(\underset{\mathrm{ijk} a b A}{\mathcal{A}})=0
$$

which in turn implies that the 1-cochain $\left\{i\left(\rho_{\mathrm{ij} a b A}^{\mathcal{A}}\right)\right\}$ is a 1-cocycle representing thus a cohomology class in $H^{1}\left(F, \mu^{*}(E) \otimes \odot^{2} \mathcal{N}_{F}^{*} \otimes \nu^{*}\left(\mathcal{E}^{*}\right)\right)$.

Next we prove items (ii) and (iii) of the Theorem for an arbitrarily chosen point $y_{0} \in Y^{\prime}$. We may assume that $y_{0}$ lies in a coordinate chart $W_{\mathrm{i}} \subset Y$ and has coordinates $\left(w_{\mathrm{i} 0}^{a}, z_{\mathrm{i} 0}^{m}\right)$. Then the alpha surface $\alpha_{y_{0}} \subset M$ is given by equations

$$
w_{\mathrm{i} 0}^{a}-\phi_{\mathrm{i}}^{a}\left(z_{\mathrm{i} 0}, t\right)=0,
$$

and, therefore, the vector field $V(t)=V^{\alpha} \partial_{\alpha}$ is tangent to $\alpha_{y_{0}}$ if and only if

$$
V^{\alpha} \partial_{\alpha} \phi_{\mathrm{i}}^{a}\left(z_{\mathrm{i} 0}, t\right)=0 .
$$

Also, just from the definition of $\mathcal{E}_{y_{0}}$ it follows that a section $h^{A}(t)$ of $\left.\mathcal{E}\right|_{\alpha_{y_{0}}}$ takes values in the subbundle $\mathcal{E}_{y_{0}}$ if and only if

$$
h^{A}(t) \lambda_{\mathrm{i} A}^{\mathcal{A}}\left(z_{\mathrm{i} 0}, t\right)=0 .
$$

Taking the partial derivative of the latter equation over $t^{\alpha}$ and using equation (7) one obtains

$$
\left(\partial_{\alpha} h^{A}(t)+\Gamma_{\alpha B}^{A}(t) h^{B}(t)\right) \lambda_{\mathrm{i} A}^{\mathcal{A}}\left(z_{\mathrm{i} 0}, t\right)=\theta_{\mathrm{i} b B} \underset{\mathcal{A}}{A}\left(z_{\mathrm{i} 0}, t\right) \frac{\partial \phi_{\mathrm{j}}^{b}\left(z_{\mathrm{i} 0}, t\right)}{\partial t^{\alpha}} h^{B}(t) .
$$

Therefore, for any vector $V \in T \alpha_{y_{0}}$,

$$
\left(\nabla_{V} h\right)^{A} \lambda_{\mathrm{i} A}^{\mathcal{A}}\left(z_{\mathrm{i} 0}, t\right)=0,
$$

which means that $\nabla_{V} h \in \mathcal{E}_{y_{0}}$ thus proving the item (ii) of the Theorem. The final item (iii) follows immediately from equation (8) which says that

$$
V^{\alpha} W^{\beta} F_{\alpha \beta A}^{B} \lambda_{\mathrm{i} B}^{\mathcal{A}}\left(z_{\mathrm{i} 0}, t\right)=0
$$

for any $V, W \in T \alpha_{y_{0}}$. This fact completes the proof of Theorem 1 .

REMARK 1. The only role of the cohomology restrictions (6) is simply to ensure that the natural map

$$
\mathcal{E} \otimes \Omega^{1} M \longrightarrow \nu_{*}^{0}\left(\mu^{*}(E) \otimes \Omega^{1} F / Y\right)
$$


is an isomorphism. Therefore the formulation of Theorem 1 can be appropriately modified.

Remark 2. In the case when the vector bundle $E$ on a twistor space $Y$ is trivial when restricted to each submanifold $X_{t} \hookrightarrow Z$, items (ii) and (iii) of Theorem 1 are exactly equivalent to the classical Ward construction [W] as formulated in [B-E], while the item (i) is equivalent to Manin's Theorem on p.125 in $[\mathrm{M}]$.

Remark 3. Often it is more suitable to work with the normal bundle $N_{t}$ of $X_{t} \hookrightarrow Y$ rather than with the subbundle $\mathcal{N}_{t} \subseteq N_{t}$. This motivates our interest in the following

Proposition 4. The statement of Theorem 1 remains true when the symbol $\mathcal{N}_{t}$ is replaced everywhere by the symbol $N_{t}$.

This Proposition is an easy consequence of the following Lemma.

Lemma 5. (i) The 1-cochain $\left\{\chi_{\mathrm{ij} b A}^{\mathcal{A}}\right\}$ with coefficients in $\mu^{*}(E) \otimes N_{F}^{*} \otimes \nu^{*}\left(\mathcal{E}^{*}\right)$ is a 1-cocycle. (ii) The 1-cochain $\left\{\rho_{\mathrm{ij} a b A}^{\mathcal{A}}\right\}$ with coefficients in $\mu^{*}(E) \otimes \odot^{2} N_{F}^{*} \otimes$ $\nu^{*}\left(\mathcal{E}^{*}\right)$ is a 1-cocycle.

Proof. We shall prove only statement (i), since the proof of (ii) is fully analogous. The equations

$$
G_{\mathrm{ik} \mathcal{B}}^{\mathcal{A}}\left(w_{\mathrm{k}}, z_{\mathrm{k}}\right)=G_{\mathrm{ijj}}^{\mathcal{A}} \mathcal{\mathcal { A }}\left(f_{\mathrm{jk}}\left(w_{\mathrm{k}}, z_{\mathrm{k}}\right), g_{\mathrm{jk}}\left(w_{\mathrm{k}}, z_{\mathrm{k}}\right)\right) G_{\mathrm{jk} \mathcal{B}}^{\mathcal{C}}\left(w_{\mathrm{k}}, z_{\mathrm{k}}\right)
$$

imply

$$
\frac{\partial G_{\mathrm{ik} \mathcal{B}}^{\mathcal{A}}}{\partial w_{\mathrm{k}}^{b}}=\frac{\partial G_{\mathrm{ij}}^{\mathcal{A} \mathcal{C}}}{\partial w_{\mathrm{j}}^{c}} \frac{\partial f_{\mathrm{jk}}^{\mathcal{C}}}{\partial w_{\mathrm{k}}^{b}} G_{\mathrm{jk} \mathcal{B}}^{\mathcal{C}}+\frac{\partial G_{\mathrm{ij} \mathcal{C}}^{\mathcal{A}}}{\partial z_{\mathrm{j}}^{m}} \frac{\partial g_{\mathrm{jk}}^{m}}{\partial w_{\mathrm{k}}^{b}} G_{\mathrm{jk} \mathcal{B}}^{\mathcal{C}}+G_{\mathrm{ij} \mathcal{C}}^{\mathcal{A}} \frac{\partial G_{\mathrm{jk} \mathcal{B}}^{\mathcal{C}}}{\partial w_{\mathrm{k}}^{b}},
$$

or

$$
\begin{aligned}
\left.\frac{\partial G_{\mathrm{ik} \mathcal{B}}^{\mathcal{A}}}{\partial w_{\mathrm{k}}^{b}}\right|_{F}= & \left.\left.\frac{\partial G_{\mathrm{ij} \mathcal{C}}^{\mathcal{A}}}{\partial w_{\mathrm{j}}^{c}}\right|_{F} F_{\mathrm{jk} b}^{c} G_{\mathrm{jk} \mathcal{B}}^{\mathcal{C}}\right|_{F}+\left.\left.G_{\mathrm{ijj}}^{\mathcal{A}}\right|_{F} \frac{\partial G_{\mathrm{jk} \mathcal{B}}^{\mathcal{C}}}{\partial w_{\mathrm{k}}^{b}}\right|_{F} \\
& +\left.\left.\left.\frac{\partial G_{\mathrm{ij} \mathcal{C}}^{\mathcal{A}}}{\partial w_{\mathrm{j}}^{c}}\right|_{F} \frac{\partial \phi_{\mathrm{j}}^{c}}{\partial z_{\mathrm{j}}^{m}} \frac{\partial g_{\mathrm{jk}}^{m}}{\partial w_{\mathrm{k}}^{b}}\right|_{F} G_{\mathrm{jk} \mathcal{B} \mathcal{B}}\right|_{F}+\left.\left.\frac{\partial G_{\mathrm{ij} \mathcal{C}}^{\mathcal{A}}}{\partial z_{\mathrm{j}}^{m}}\right|_{F} \frac{\partial g_{\mathrm{jk}}^{m}}{\partial w_{\mathrm{k}}^{b}} G_{\mathrm{jk} \mathcal{B}}^{\mathcal{C}}\right|_{F}
\end{aligned}
$$

where the symbol $\left.\right|_{F}$ means the restriction from $Y \times M$ to $F$, i.e. the substitution of functions $\phi_{\mathrm{k}}\left(z_{\mathrm{k}}, t\right)$ for coordinates $w_{\mathrm{k}}$. Taking into account equation (4), one therefore obtains

$$
\delta\left\{\left.\frac{\partial G_{\mathrm{ij} \mathcal{B}}^{\mathcal{A}}}{\partial w_{\mathrm{j}}^{b}}\right|_{w_{\mathrm{j}}=\phi_{\mathrm{j}}} \lambda_{\mathrm{j} A}^{\mathcal{B}}\right\}=\left\{\left.\left.\frac{\partial\left(\left.G_{\mathrm{ij}}^{\mathcal{A}}\right|_{F}\right)}{\partial z_{\mathrm{j}}^{m}} \frac{\partial g_{\mathrm{jk}}^{m}}{\partial w_{\mathrm{k}}^{b}}\right|_{F} G_{\mathrm{jk} \mathcal{B}}^{\mathcal{C}}\right|_{F}\right\} .
$$


In a similar way, one can use equations (4) and

$$
g_{\mathrm{ik}}\left(w_{\mathrm{k}}, z_{\mathrm{k}}\right)=g_{\mathrm{ij}}\left(f_{\mathrm{jk}}\left(w_{\mathrm{k}}, z_{\mathrm{k}}\right), g_{\mathrm{jk}}\left(w_{\mathrm{k}}, z_{\mathrm{k}}\right)\right)
$$

to show that

$$
\delta\left\{\left.\frac{\partial \lambda_{\mathrm{i} A}^{\mathcal{A}}}{\partial z_{\mathrm{i}}^{m}} \frac{\partial g_{\mathrm{ij}}^{m}}{\partial w_{\mathrm{j}}^{b}}\right|_{F}\right\}=\left\{\left.\left.\frac{\partial\left(\left.G_{\mathrm{ij} \mathcal{C}}^{\mathcal{A}}\right|_{F}\right)}{\partial z_{\mathrm{j}}^{m}} \frac{\partial g_{\mathrm{jk}}^{m}}{\partial w_{\mathrm{k}}^{b}}\right|_{F} G_{\mathrm{jk} \mathcal{B}}^{\mathcal{C}}\right|_{F}\right\} .
$$

Finally, the last equation together with (11) imply that

$$
\delta\left\{\left.\frac{\partial G_{\mathrm{ij} \mathcal{B}}^{\mathcal{A}}}{\partial w_{\mathrm{j}}^{b}}\right|_{F} \lambda_{\mathrm{j} A}^{\mathcal{B}}-\left.\frac{\partial \lambda_{\mathrm{i} A}^{\mathcal{A}} \frac{\partial g_{\mathrm{ij}}^{m}}{\partial z_{\mathrm{i}}^{m}}}{\partial w_{\mathrm{j}}^{b}}\right|_{F}\right\}=0
$$

which proves Lemma 5.

REMARK 4. If $H^{0}\left(X_{t},\left.E\right|_{X_{t}} \otimes \mathcal{N}_{t}^{*}\right) \neq 0$, but the other conditions of Theorem 1 are satisfied, then the induced vector bundle $\mathcal{E}$ comes equipped not with a unique connection $\nabla$, but with a whole family of induced connections $\{\nabla\}$ such that for any $t \in M$ and any pair of connections, $\nabla_{1}$ and $\nabla_{2}$, from the family one has

$$
\left.\nabla_{1}\right|_{t}-\left.\nabla_{2}\right|_{t} \in j\left(H^{0}\left(X_{t},\left.E\right|_{X_{t}} \otimes \mathcal{N}_{t}^{*}\right)\right) \otimes \mathcal{E}_{t}^{*},
$$

where

$$
j: H^{0}\left(X_{t},\left.E\right|_{X_{t}} \otimes \mathcal{N}_{t}^{*}\right) \longrightarrow \mathcal{E}_{t} \otimes \Omega_{t}^{1} M
$$

is a natural map constructed with the help of the Kodaira map $k: T_{t} M \longrightarrow H^{0}\left(X_{t}, \mathcal{N}_{t}\right)$. Indeed, in this case the cochain $\left\{\theta_{\mathrm{i} b A}^{\mathcal{A}}\right\}$ is determined by equation (6) up to a global section of $\mu^{*}(E) \otimes \mathcal{N}_{F}^{*} \otimes \nu^{*}\left(\mathcal{E}^{*}\right)$. The same arguments as before show that for any connection from the family $\{\nabla\}$ items (i)-(iii) of Theorem 1 remain true.

REMARK 5. Let $X$ be a rational curve in a complex surface $Y$ with normal bundle $\mathcal{O}(2)$. Then the Kodaira moduli space $M$ parametrizing holomorphic deformation of $X$ inside $Y$ exists and is a complex 3-fold which, as shown by Hitchin $[\mathrm{H}]$, comes equipped with an induced Einstein-Weyl structure. Assume that the canonical bundle $\kappa$ on $Y$ admits a cubic root. Then the bundle

$$
E=\kappa^{-\frac{1}{4}}
$$

is such that for any $t \in M$,

$$
\left.E\right|_{X_{t}}=\mathcal{O}(1)
$$

Then all the conditions of Theorem 1 are satisfied, and, therefore, the in- 
duced rank 2 vector bundle $\mathcal{E} \rightarrow M$ comes equipped canonically with an induced connection $\nabla$. The items (i)-(iii) of Theorem 1 imply that $\nabla$ is nothing but a spinor version of the Einstein-Weyl connection on $M$.

2.3. Twistor interpretation of $J^{1} \mathcal{E}^{*}$. Elements of the induced vector bundle $\mathcal{E}$ over a point $t \in M$ have a clear twistor interpretation - they correspond to global sections of $E$ over the associated submanifold $X_{t}$. Often, however, we are also interested in elements of $J^{1} \mathcal{E}$ (in field theory, for example, Lagrangians are usually constructed from first jets of sections of vector bundles). It is the purpose of this subsection to find a (surprisingly simple!) twistor decoding of $J^{1} \mathcal{E}^{*}$ and, as a by-product, to prove a stronger version of Theorem 1. To simplify notation, we assume in this and the subsequent section that for any submanifold $X \hookrightarrow Y$ under consideration the map

$$
H^{0}(X, N) \otimes N \longrightarrow N
$$

is an epimorphism, i.e. $N=\mathcal{N}$. This assumption is by no means crucial for the conclusions drawn below.

Let $\left(X, \mathcal{O}_{X}\right)$ be a complex submanifold of a complex manifold $\left(Y, \mathcal{O}_{Y}\right)$ and $E$ a holomorphic vector bundle on $Y$. Assume that at any $x \in X$ the stalk of $\left.E\right|_{X}$ is spanned by germs of global sections, i.e. there is an epimorphism

$$
\mathcal{E}_{0} \otimes_{\mathrm{C}} \mathcal{O}_{X} \stackrel{i_{1}}{\longrightarrow} E_{X} \longrightarrow 0,
$$

where $\mathcal{E}_{0} \equiv H^{0}\left(X, E_{X}\right)$ and $\left.E_{X} \equiv E\right|_{X}$. Consider the restriction of the dual of $E$ to the first order infinitesimal neighbourhoood of $X$ in $Y$ which, as an $\mathcal{O}_{X}^{(1)}$-module, has the following extension structure

$$
\left.0 \longrightarrow E_{X}^{*} \otimes N^{*} \stackrel{i_{2}}{\longrightarrow} E^{*}\right|_{X^{(1)}} \longrightarrow E_{X}^{*} \longrightarrow 0 .
$$

Define the composition

$$
\mathrm{i}_{3}:\left.E_{X}^{*} \otimes N^{*} \stackrel{i_{4}}{\longrightarrow} E_{X}^{*} \otimes N^{*} \oplus E_{X}^{*} \otimes N^{*} \stackrel{*_{1}^{*} \otimes i d \otimes i_{2}}{\longrightarrow} \mathcal{E}_{0}^{*} \otimes N^{*} \oplus E^{*}\right|_{X^{(1)}}
$$

where

$$
\begin{aligned}
i_{4}: E_{X}^{*} \otimes N^{*} & \longrightarrow E_{X}^{*} \otimes N^{*} \oplus E_{X}^{*} \otimes N^{*} \\
f & \longrightarrow f \oplus(-f) .
\end{aligned}
$$

The quotient sheaf of $\mathcal{O}_{X}^{(1)}$-modules,

$$
\mathfrak{E}_{1}^{*} \equiv\left[\left.\mathcal{E}_{0}^{*} \otimes N^{*} \oplus E^{*}\right|_{X^{(1)}}\right] / i_{3}\left[E_{X}^{*} \otimes N^{*}\right]
$$

fits into the commutative diagram 


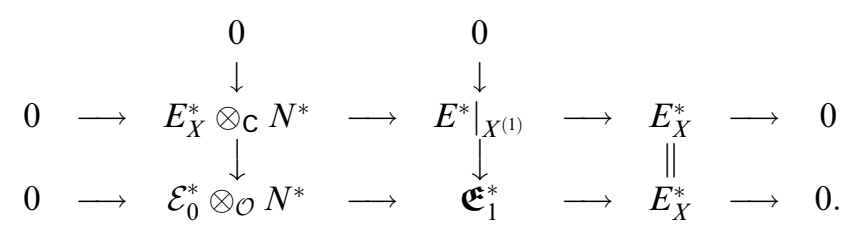

Next define another $\mathcal{O}_{X}^{(1)}$-module $\mathfrak{E}_{2}^{*}$ by the following commutative diagram



Finally from the exact sequence

$$
0 \longrightarrow \mathcal{E}_{0}^{*} \otimes N^{*} \oplus \mathcal{E}_{0}^{*} \otimes N^{*} \stackrel{h}{\longrightarrow} \mathfrak{E}_{1}^{*} \oplus \mathfrak{E}_{2}^{*} \stackrel{g}{\longrightarrow} E_{X}^{*} \oplus E_{X}^{*} \longrightarrow 0,
$$

we obtain a "difference" sheaf of $\mathcal{O}_{X}^{(1)}$-modules

$$
\mathfrak{E}_{X}^{*}=g^{-1}\left(\text { diagonal in } \oplus E_{X}^{*}\right) / h\left(\text { diagonal in } \oplus \mathcal{E}_{0}^{*} \otimes N^{*}\right) \text {. }
$$

It is clear that $\mathfrak{E}_{X}^{*}=\left[\mathfrak{E}_{1}^{*}\right]-\left[\mathfrak{E}_{2}^{*}\right]$ in $\operatorname{Ext}_{\mathcal{O}_{X}^{(1)}}^{1}\left(E_{X}^{*}, \mathcal{E}_{0}^{*} \otimes N^{*}\right)$. The consequences of these constructions are the following Lemma and Proposition.

Lemma 6. $\mathfrak{E}_{X}^{*}$ is locally free on $X$.

Proof. A sufficiently small tubular neighbourhood of $X$ in $Y$ can be covered by the Leray coordinate charts $\left\{W_{\mathrm{i}}\right\}$ with local coordinate functions $\left(w_{\mathrm{i}}^{a}, z_{\mathrm{i}}^{m}\right)$ such that the intersection $X \cap W_{\mathrm{i}}$ is given by $w_{\mathrm{i}}^{a}=0$. Then a choice of trivialisation of $E$ over each $W_{\mathrm{i}}$ induces a trivialisation

$$
\gamma_{\mathrm{i}}:\left.\left.\left.\mathfrak{E}_{1}^{*}\right|_{W_{\mathrm{i}} \cap X} \longrightarrow \mathcal{E}_{0}^{*} \otimes N^{*}\right|_{W_{\mathrm{i}} \cap X} \oplus E\right|_{W_{\mathrm{i}} \cap X} .
$$

Keeping the notation of the proof of Theorem 1, one may easily find the transition matrix of such trivialisations

$$
\gamma_{\mathrm{i}} \circ \gamma_{\mathrm{j}}^{-1}=\left(\begin{array}{cc}
\left.\delta_{A}^{B} \frac{\partial f_{\mathrm{ij}}^{b}}{\partial w_{\mathrm{j}}^{a}}\right|_{X} & \left.\lambda_{\mathrm{j} A}^{\mathcal{C}} \frac{\partial G_{\mathrm{ij} \mathcal{C}}^{\mathcal{B}}}{\partial w_{\mathrm{j}}^{a}}\right|_{X} \\
0 & \left.G_{\mathrm{ij} \mathcal{A}}^{\mathcal{B}}\right|_{X}
\end{array}\right) .
$$

The analogous transition matrix for $\mathfrak{E}_{2}^{*}$ has the form

$$
\left(\begin{array}{ccc}
\left.\delta_{A}^{B} \frac{\partial f_{\mathrm{ij}}^{b}}{\partial w_{\mathrm{j}}^{a}}\right|_{X} & \left.\frac{\partial \lambda_{\mathrm{i} A}^{\mathcal{B}}}{\partial z_{\mathrm{i}}^{m}} \frac{\partial g_{\mathrm{ij}}^{m}}{\partial w_{\mathrm{j}}^{a}}\right|_{X} \\
0 & \left.G_{\mathrm{ij} \mathcal{A}}^{\mathcal{B}}\right|_{X}
\end{array}\right) .
$$

Neither of these matrices match up on triple intersections, reflecting the fact 
that neither $\mathfrak{E}_{1}^{*}$ nor $\mathfrak{E}_{2}^{*}$ are locally free. By the very definition of $\mathfrak{E}_{X}^{*}$, its transition matrix has the form

$$
H_{\mathrm{ij}}=\left(\begin{array}{cc}
\left.\left.\delta_{A}^{B} \frac{\partial f_{\mathrm{ij}}^{b}}{\partial w_{\mathrm{j}}^{a}}\right|_{X} \quad \lambda_{\mathrm{j} A}^{\mathcal{C}} \frac{\partial G_{\mathrm{ij} \mathcal{C}}^{\mathcal{B}}}{\partial w_{\mathrm{j}}^{a}}\right|_{X}-\left.\frac{\partial \lambda_{\mathrm{i} A}^{\mathcal{B}}}{\partial z_{\mathrm{i}}^{m}} \frac{\partial g_{\mathrm{ij}}^{m}}{\partial w_{\mathrm{j}}^{a}}\right|_{X} \\
0 & \left.G_{\mathrm{ij} \mathcal{A}}^{\mathcal{B}}\right|_{X}
\end{array}\right)
$$

By Lemma (5), the 1-cochain $\left\{\left.\lambda_{\mathrm{j} A}^{\mathcal{C}} \frac{\partial G_{\mathrm{ij}, A}^{\mathcal{C}}}{\partial w_{\mathrm{j}}^{a}}\right|_{X}-\left.\frac{\partial \lambda_{\mathrm{i} A}^{\mathcal{B}}}{\partial z_{\mathrm{i}}^{m}} \frac{\partial g_{\mathrm{ij}}^{m}}{\partial w_{\mathrm{j}}^{a}}\right|_{X}\right\}$ is a cocycle, which implies that the matrices $H_{\mathrm{ij}}$ satisfy on triple intersections $W_{\mathrm{i}} \cap W_{\mathrm{j}} \cap W_{\mathrm{k}}$ the condition $H_{\mathrm{ik}}=H_{\mathrm{ij}} H_{\mathrm{jk}}$ which is necessary and sufficient for the sheaf $\mathfrak{E}_{X}^{*}$ to be locally free on $X$. This fact completes the proof.

By construction, the vector bundle $\mathfrak{E}_{X}$ fits into an exact sequence

$$
0 \longrightarrow E_{X} \longrightarrow \mathfrak{E}_{X} \longrightarrow \mathcal{E}_{0} \otimes N \longrightarrow 0 \text {. }
$$

THEOREM 7. Let $\left\{X_{t} \hookrightarrow Y \mid t \in M\right\}$ be a family of compact submanifolds and $E \rightarrow Y$ an $M$-homogeneous vector bundle. Then, for each $t \in M$, there is a commutative diagram of vector spaces



with rows exact.

Proof. Keeping the notation of subsection 2.2, define the map

$$
\begin{aligned}
& \left(J^{1} \mathcal{E}^{*}\right)_{t}^{*} \longrightarrow H^{0}\left(X_{t}, \mathfrak{E}_{X_{t}}\right) \\
& {\left[\begin{array}{l}
V^{\alpha B} \\
V^{B}
\end{array}\right] \longrightarrow\left[\begin{array}{c}
V^{\alpha B} \partial_{\alpha} \phi_{\mathrm{i}}^{a} \\
V^{\alpha B} \partial_{\alpha} \lambda_{\mathrm{i} B}^{\mathcal{A}}+V^{B} \lambda_{\mathrm{i} B}^{\mathcal{A}}
\end{array}\right]}
\end{aligned}
$$

By equation (5) and the explicit description of the transition matrix of $\mathfrak{E}_{X_{t}}$ given in the proof of Lemma 6, this map is well-defined and has, evidently, the properties stated by the commutative diagram above.

Therefore, with a weakly $M$-uniform vector bundle $E$ on a twistor space $Y$ and any submanifold $X_{t} \hookrightarrow Y$ of the family $F \hookrightarrow Y \times M$ there is a naturally associated element $\chi_{t} \in H^{1}\left(X_{t}, E_{X_{t}} \otimes N_{t}^{*}\right) \otimes\left(H^{0}\left(X_{t}, E_{X_{t}}\right)\right)^{*}$ which is the obstruction to a global splitting of the exact sequence

$$
0 \longrightarrow E_{X_{t}} \longrightarrow \mathfrak{E}_{X_{t}} \longrightarrow N_{t} \otimes H^{0}\left(X_{t}, E_{X_{t}}\right) \longrightarrow 0 .
$$

A vector bundle $E \rightarrow Y$ is said to be $M_{(1)}$ - uniform if it is weakly $M$-uniform 
and $\chi_{t}=0$ for all $t \in M$ (the subscript in the symbol $M_{(1)}$ is used to remind us that certain assumptions about $\left.E\right|_{X_{t}^{(1)}}$ are now in force).

Treating the triple $\left(\pi_{1}^{*}(E), F \hookrightarrow Y \times M\right), \pi_{1}$ being a natural projection $Y \times M \rightarrow Y$, along the same lines as the triple $(E, X \hookrightarrow Y)$ above, one constructs a locally free sheaf $\mathfrak{E}_{F}$ on $F$ which fits into an exact sequence

$$
0 \longrightarrow \mu^{*}(E) \stackrel{i}{\longrightarrow} \mathfrak{E}_{F} \longrightarrow N_{F} \otimes \nu^{*}(\mathcal{E}) \longrightarrow 0
$$

and has the property that the following diagram

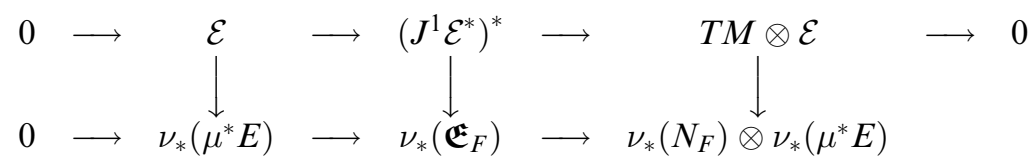

is commutative.

If $E$ is an $M_{(1)}$-uniform vector bundle on $Y$, then the extension (14) splits, i.e. admits a morphism $s: \mathfrak{E}_{F} \rightarrow \mu^{*}(E)$ such that $s \circ i=i d$. Any such splitting induces, via the commutative diagram (15), a map $s^{\prime}:\left(J^{1} \mathcal{E}^{*}\right)^{*} \rightarrow \mathcal{E}$, which splits the extension

$$
0 \longrightarrow \mathcal{E} \stackrel{j}{\longrightarrow}\left(J^{1} \mathcal{E}^{*}\right)^{*} \longrightarrow \mathcal{E} \otimes T M \longrightarrow 0,
$$

i.e. satisfies $s^{\prime} \circ j=$ id. It is well known [M] that any such splitting is equivalent to a linear connection on $\mathcal{E}$. A linear connection on $\mathcal{E}$ which comes from a splitting of (14) is said to be an induced connection. If $H^{0}\left(X_{t}, E_{X_{t}} \otimes N_{t}^{*}\right)=0$ for all $t \in M$, then there is only one splitting of (14) and hence a unique induced connection on $\mathcal{E}$. Of course, this is the same creature that was studied in Theorem 1. In general, since the set of all splittings of (14) is a principal homogeneous space for the group $H^{0}\left(F, \mu^{*}(E) \otimes N_{F}^{*} \otimes \nu^{*}\left(\mathcal{E}^{*}\right)\right)$, we obtain the following strengthening of Theorem 1

THEOREM 8. Let $F \hookrightarrow Y \times M$ be a family of compact complex submanifolds and $E$ an $M_{(1)}$-uniform vector bundle on $Y$. Then the induced vector bundle $\mathfrak{E}$ on $M$ comes equipped canonically with a family $\{\nabla\}$ of induced connections such that

(a) if $\nabla_{1}$ and $\nabla_{2}$ are in $\{\nabla\}$, then $\nabla_{1}-\nabla_{2} \in H^{0}\left(M, \nu_{*}\left(\mu^{*} E \otimes N_{F}\right) \otimes \mathfrak{E}^{*}\right)$;

(b) the items (i)-(iii) of Theorem 1 are true for any $\nabla \in\{\nabla\}$

2.4. Inverse construction. Let $F \hookrightarrow Y \times M$ be a family of compact complex submanifolds with $Y=\cup_{t \in M}$ and let $\mathcal{E}$ be a vector bundle on $M$ with a linear connection $\nabla$. Assume that $\nu^{*}(\mathcal{E})$ fits into an exact sequence

$$
0 \longrightarrow \mathfrak{T}_{F} \longrightarrow \nu *(\mathcal{E}) \longrightarrow \mathfrak{S}_{F} \longrightarrow 0
$$

for some vector bundles $\mathfrak{S}_{F}$ and $\mathfrak{T}_{F}$ on $F$. Consider the composition 


$$
\nabla_{F / Y}: \nu^{*}\left(\mathcal{E}^{*}\right) \stackrel{\nu^{*}(\nabla)}{\longrightarrow} \nu^{*}\left(\mathcal{E}^{*}\right) \otimes \nu^{*}\left(\Omega^{1} M\right) \longrightarrow \nu^{*}\left(\mathcal{E}^{*}\right) \otimes \Omega^{1} F / Y
$$

and assume that $\nabla_{F / Y}$ is flat when restricted to the subbundle $\mathfrak{S}_{F}^{*} \subset \nu^{*}\left(\mathcal{E}^{*}\right)$. Denote by $E_{F}$ the $\mu^{-1}\left(\mathcal{O}_{Y}\right)$-module of $\nabla_{F / Y}$-horizontal sections of $\mathfrak{S}_{F}^{*}$. Assuming that the fibres of $\mu$ are simply connected, we obtain a locally free sheaf $E=\left(\mu_{*} E_{F}\right)^{*}$ on the twistor space $Y$ with $\operatorname{rank} E^{*}=\operatorname{rank} \mathfrak{S}_{F}^{*}$. Assuming also that $\nu_{*}\left(\mathfrak{T}_{F}\right)=\nu_{*}^{1}\left(\mathfrak{T}_{F}\right)=0$, we conclude that $\nu_{*}\left(\mu^{*} E\right)=\mathcal{E}$, i.e. $\mathcal{E} \rightarrow M$ is a vector bundle induced from the weakly $M$-uniform vector bundle $E \rightarrow Y$. To complete this inverse construction we have to prove the following

Proposition 9. The bundle $E=\left(\mu_{*} E_{F}\right)^{*}$ is an $M_{(1)}$-uniform vector bundle such that $\nabla$ is an induced connection on $\mathcal{E}$.

A SKETCH OF THE PROOF. We have to prove that there exists a splitting of the extension (14) such that the associated induced connection on $\mathcal{E}$ is exactly the original linear connection $\nabla$. Let $g$ be a germ in the stalk of $N_{F} \otimes \nu^{*}(\mathcal{E})$ at a point $p \in F$. One can use the epimorphism

$$
\nu^{*}(T M \otimes \mathcal{E}) \stackrel{i_{1}}{\longrightarrow} N_{F} \otimes \nu^{*}(\mathcal{E}) \longrightarrow 0
$$

and the monomorphism $i_{\nabla}: \nu^{*}(T M \otimes \mathcal{E}) \longrightarrow \nu^{*}\left(J^{1} \mathcal{E}^{*}\right)^{*}$ associated with $\nu^{*}(\nabla)$ to define the subset $i_{\nabla} \circ i_{1}^{-1}(g) \subset \nu^{*}\left(J^{1} \mathcal{E}^{*}\right)^{*}$. Then, using the composition

$$
i_{2}: \nu^{*}\left(J^{1} \mathcal{E}^{*}\right)^{*} \longrightarrow \nu^{*}\left(\nu_{*} \mathfrak{E}_{F}\right) \stackrel{e v_{p}}{\longrightarrow} \mathfrak{E}_{F},
$$

where $e v_{p}$ is the natural map of "evaluation of a global section at p", one finally obtains the subset $\triangle(g)=i_{2} \circ i_{\nabla} \circ i_{1}^{-1}(g)$ of the stalk of $\mathfrak{E}_{F}$ at $p$. Since the diagram (15) is commutative, the image of $\triangle(g)$ under the epimorphism $\mathfrak{E}_{F} \rightarrow N_{F} \otimes \nu^{*}(\mathcal{E})$ is the original germ $g$. Thus if one can show that the set $\triangle(g)$ consists of only one element, then one would obtain a genuine splitting of the extension (14) thus proving the Proposition. This final check can be easily made with the help of the local coordinate descriptions of $\mathfrak{E}_{F}$ and all the maps involved in the construction (see subsections 2.2 and 2.3).

\section{Example.}

3.1. Conic structures and conic connections. Let $M$ be a complex $n$-dimensional manifold and $d \leq n$ an integer. A $d$-conic structure on $M$ is a closed complex submanifold $i: \mathcal{F} \hookrightarrow \mathrm{G}_{M}\left(n-d ; \Omega^{1} M\right)$ such that the projection $\nu: \mathcal{F} \rightarrow M$ is a holomorphic submersion [M]. Thus $\mathcal{F} \rightarrow M$ is a fibration with fibres isomorphic to an algebraic subspace in the Grassmanian $\mathrm{G}\left(n-d, \mathrm{C}^{n}\right)$; any point $p \in \mathcal{F}$ can be identified with an $(n-d)$-dimensional subspace $p \subset \Omega_{\nu(p)} M$ and hence with a $d$-dimensional subspace $p^{\perp} \subset T_{\nu(p)} M$. A conic connection on $\mathcal{F}$ is, by definition, a holomorphic rank $d$ distribution 
$\mathcal{D} \subset T \mathcal{F}$ such that, at each $p \in \mathcal{F}, d \nu(D)$ is exactly $p^{\perp} \subset T_{\nu(p)} M$. A conic connection is said to be integrable if it is integrable as a distribution.

Let $\mathfrak{U}$ be the tautological vector bundle on the relative Grassmanian $\mathrm{G}_{M}\left(n-d, \Omega^{1} M\right)$ and $\mathfrak{V}$ the dual of the quotient $\sigma^{*}\left(\Omega^{1} M\right) / \mathfrak{U}$, where $\sigma: \mathrm{G}_{M}\left(n-d, \Omega^{1} M\right) \rightarrow M$ is the natural projection. It is clear that $\mathfrak{V} \subset \sigma^{*}(T M)$ and hence $i^{*}(\mathfrak{V}) \subset \nu^{*}(T M)$. Then, defining

$$
\tilde{T} \mathcal{F}=d \nu^{-1}\left(i^{*}(\mathfrak{V})\right),
$$

one may regard a conic connection on $\mathcal{F}$ as a splitting of the exact sequence

$$
0 \longrightarrow T \mathcal{F} / M \longrightarrow \tilde{T} \mathcal{F} \stackrel{d \nu}{\longrightarrow} i^{*}(\mathfrak{V}) \longrightarrow 0,
$$

where $T \mathcal{F} / M$ is the sheaf of $\nu$-vertical vector fields on $\mathcal{F}$. It is therefore natural to call $\nu_{*}\left(i^{*}\left(\mathfrak{V}^{*}\right) \otimes T \mathcal{F} / M\right)$ the sheaf of conic connection coefficients.

3.2. Twistor space of an exotic $G_{3}$-structure. Let $\mathrm{S}$ be a standard 2-dimensional representation space of $G L(2, \mathrm{C})$. Then $G L(2, \mathrm{C})$ naturally acts on the symmetric tensor product $\odot^{3} \mathrm{~S}$. If $\rho: G L(2, \mathrm{C}) \rightarrow G L(4, \mathrm{C})$ is the associated representation, one may define a subgroup $G_{3}=\rho(G L(2, \mathrm{C}))$ of $G L(4, \mathrm{C})$. Let $M$ be a complex 4-manifold and $\pi: L^{*} M \rightarrow M$ the holomorphic coframe bundle whose fibres $L_{t}^{*} M=\pi^{-1}(t)$ consist of all C-linear isomorphisms $e: \mathrm{C}^{4} \rightarrow \Omega_{t}^{1} M$. The space $L^{*} M$ is naturally a principal right $G L(4, \mathrm{C})$-bundle, where the right action $R_{g}: L^{*} M \rightarrow L^{*} M$ is given by $R_{g}(e)=e \circ g$. A $G_{3}$-structure on $M$ is, by definition, is a principal subbundle of $L^{*} M$ with the group $G_{3}$. It is clear that a $G_{3}$-structure is equivalent to a local factorization of the tangent bundle into the symmetric cube

$$
T M=\odot^{3} \mathcal{S}
$$

of a locally defined vector bundle $\mathcal{S}$ of rank 2 . Though such a vector bundle may fail to exist on the whole of $M$, the projectivised vector bundle $\mathrm{P}_{\mathrm{M}}(\mathcal{S})$ is well-defined globally. Since we are interested in this paper in local properties of $G_{3}$-structures, we assume from now on that $M$ is a complex 4-manifold with $G_{3}$-structure such that $\mathcal{S}$ exists on the whole of $M$; it is called a spinor bundle on $M$. A linear connection $\nabla$ on $\mathcal{S}$ is called a spinor connection on $M$. It is clear that any spinor connection on $M$ induces, via the isomorphism $T M=\odot^{3} \mathcal{S}$, an affine connection with holonomy in $G_{3}$; moreover, any affine connection on $M$ with holonomy in $G_{3}$ arises, at least locally, in this way. By a torsion tensor of a spinor connection we mean the torsion tensor of the associated affine connection.

There is a canonical injective bundle map, $i: \mathrm{P}_{M}\left(\mathcal{S}^{*}\right) \rightarrow \mathrm{G}_{M}\left(2, \Omega^{1} M\right)$, which can be unambiguously characterised by the isomorphism $i^{*}(\mathfrak{U})=\nu *\left(\mathcal{S}^{*}\right)(-2) \equiv \nu^{*}\left(\mathcal{S}^{*}\right) \otimes \mathcal{O}_{\mathcal{F}}(-2)$, where $\mathcal{O}_{\mathcal{F}}(-2)=\left[\mathcal{O}_{\mathcal{F}}(-1)\right]^{\otimes 2}$ and $\mathcal{O}_{F}(-1)$ stands for the tautological sheaf on $\mathrm{P}_{M}\left(\mathcal{S}^{*}\right)$. Thus $\mathrm{P}_{M}\left(\mathcal{S}^{*}\right)$ is natu- 
rally a 2-conic structure $\mathcal{F}$ on $M$. Since $i^{*}(\mathfrak{V})=\nu^{*}\left(\mathcal{S} \otimes\left(\Lambda^{2} \mathcal{S}\right)^{\otimes 2}\right)(-2)$ and $T \mathcal{F} / M=\nu^{*}\left(\Lambda^{2} \mathcal{S}^{*}\right)(2)$, the conic connection coefficient sheaf on $\mathcal{F}$ is isomorphic to

$$
\nu_{*}^{0}\left(T \mathcal{F} / M \otimes i^{*}(\mathfrak{V})\right)=\odot^{5} \mathcal{S}^{*} \otimes \Lambda^{2} \mathcal{S} \oplus \odot^{3} \mathcal{S}^{*}
$$

A projective connection on $\mathcal{F}=\mathrm{P}_{M}\left(\mathcal{S}^{*}\right)$ determines a splitting of the exact sequence

$$
0 \longrightarrow T \mathcal{F} / M \longrightarrow T \mathcal{F} \stackrel{d \nu}{\longrightarrow} \nu^{*}(T M) \longrightarrow 0,
$$

that is a morphism $\gamma: \nu^{*}(T M) \rightarrow T \mathcal{F}$ such that $d \nu \circ \gamma=i d$. Then, restricting $\gamma$ to the subsheaf $\mathfrak{V} \subset \nu^{*}(T M)$, one gets a conic connection on $\mathcal{F}$. From the exact sequence which relates coefficient sheaves of projective and 2-conic connections,

$$
0 \longrightarrow \nu^{*}\left(\mathcal{S}^{*} \otimes \Lambda^{2} \mathcal{S}^{*}\right) \longrightarrow T \mathcal{F} / M \otimes \nu^{*}\left(\Omega^{1} M\right) \longrightarrow T \mathcal{F} / M \otimes i^{*}(\mathfrak{V}) \longrightarrow 0,
$$

it is clear that the map

$\left\{\right.$ projective connections on $\left.\mathrm{P}_{\mathrm{M}}\left(\mathcal{S}^{*}\right)\right\} \longrightarrow\{$ conic connections on $\mathcal{F}$ \}

is surjective with its kernel given by sections of $\mathcal{S}^{*} \otimes \Lambda^{2} \mathcal{S}^{*}$. Hence the kernel of the surjection

$$
\{\text { linear connections on } \mathcal{S}\} \stackrel{\mathrm{pr}}{\longrightarrow}\{\text { conic connections on } \mathcal{F}\}
$$

consists of arbitrary sections of $\mathcal{S}^{*} \otimes \Lambda^{2} \mathcal{S}^{*} \oplus \odot^{3} \mathcal{S}^{*}$. Using this freedom together with (16), it is not hard to check that there exists a unique 2-conic connection $\mathcal{D}$ on $\mathcal{F}$, called the distinguished 2-conic connection, such that the set $\operatorname{pr}^{-1}(\mathcal{D})$ contains a (necessarily unique) linear connection $\nabla$ whose torsion tensor is a section of $\odot^{7} \mathcal{S}^{*} \otimes\left(\Lambda^{2} \mathcal{S}\right)^{\otimes 2} \subset T M \otimes \Omega^{2} M$. Then this section is nothing but the invariant torsion [Brl] of the $G_{3}$-structure. The vanishing of this section is a necessary and sufficient condition for the $G_{3}$-structure to admit a torsion-free affine connection.

Proposition 10. [Br2] $A G_{3}$-structure on a complex 4-fold $M$ admits a torsion-free affine connection if and only if the distinguished 2-conic connection on $\mathcal{F}_{2}$ is integrable.

Thus, with any torsion-free $G_{3}$-structure on a sufficiently "small" 4-fold $M$ there is naturally associated a double fibration

$$
Y \stackrel{\mu}{\longleftarrow} \mathcal{F} \stackrel{\nu}{\longrightarrow} M
$$

where $Y$ is a 3-dimensional complex manifold (called the twistor space) parametrising leaves of the integrable distribution $\mathcal{D}$. It is not difficult to show that the composition 


$$
\nabla_{\mathcal{F} / Y}: \nu^{*}\left(\mathcal{S}^{*}\right) \stackrel{\nu^{*}(\nabla)}{\longrightarrow} \nu^{*}\left(\mathcal{S}^{*} \otimes \Omega^{1} M\right) \longrightarrow \nu^{*}\left(\mathcal{S}^{*}\right) \otimes \Omega^{1} \mathcal{F} / Y
$$

preserves the subbundle $\mathcal{O}_{\mathcal{F}}(-1) \subset \nu^{*}\left(\mathcal{S}^{*}\right)$. Moreover, an analysis of the curvature tensor of $\nabla$ through the Bianchi identities (cf. [Br2]) implies that $\nabla_{\mathcal{F} / Y}$ is actually flat on $\mathcal{O}_{\mathcal{F}}(-1)$. Thus one may define a holomorphic line bundle

$$
E^{*}=\left.\operatorname{Ker} \nabla_{\mathcal{F} / Y}\right|_{\mathcal{O}_{\mathcal{F}(-1)}}
$$

on $Y$ whose restriction to each rational curve $\mu \circ \nu^{-1}(t), t \in M$, is isomorphic to $\mathcal{O}(-1)$. Then Proposition 9 implies that both the spinor bundle $\mathcal{S}$ and the torsion-free affine connection $\nabla$ are induced, in the sense of Theorem 1, from the vector bundle $E \rightarrow Y$. The manifold $Y$ comes equipped with a 4-parameter family of rational curves $X_{t}=\mu \circ \nu^{-1}(t)$ with the normal bundle $\mathrm{C}^{2} \otimes \mathcal{O}(2)$. Therefore, the family $\left\{X_{t} \hookrightarrow Y \mid t \in M\right\}$ is not complete in the Kodaira sense making the hopes of restoring $M$ with the help of the Kodaira relative deformation theorem gloomy. However, the prospects of twistor methods improve when one takes into account the complex contact structure [Br2] canonically induced on $Y$. There is a canonical embedding

$$
j: \mathrm{P}_{M}\left(\mathcal{S}^{*}\right) \rightarrow \mathrm{P}_{M}\left(\Omega^{1} M\right)
$$

which can be unambiguously characterised by the isomorphism $j^{*}\left(\mathcal{O}_{\mathrm{P}_{M}\left(\Omega^{1} M\right)}(1)\right)=\mathcal{O}_{\mathrm{P}_{M}\left(\mathcal{S}^{*}\right)}(3)$. As shown in [Me2] in a more general situation, the contact line bundle $L$ on $Y$ is nothing but $\left.\operatorname{Ker} \nabla_{\mathcal{F} / Y}\right|_{j^{*}(\tilde{L})}$, where $\tilde{L} \simeq \mathcal{O}_{\mathrm{P}_{M}\left(\Omega^{1} M\right)}(1)$ is the canonical contact line bundle on $\mathrm{P}_{M}\left(\Omega^{1} M\right)$. Therefore, $L=E^{\otimes 3}$. Since $h^{0}\left(X_{t},\left.E^{\otimes 3}\right|_{X_{t}}\right)=h^{0}\left(\mathrm{CP}^{1}, \mathcal{O}(3)\right)=4$, the family $\left\{X_{t} \hookrightarrow Y \mid t \in M\right\}$ is complete as a family of compact Legendre submanifolds of $Y \quad[\mathrm{Br} 2, \mathrm{Me} 1, \mathrm{Me} 2]$. Therefore, given a Legendre submanifold $X=\mathrm{CP}^{1} \hookrightarrow Y$ of a complex contact manifold $Y$ with contact line bundle $L$ such that $L_{X}=\mathcal{O}(3)$, one may first construct a 4-dimensional Legendre moduli space $M$ and then, shrinking $Y$ as necessary and then choosing any $E$ such that $E^{\otimes 3}=L$, one may apply Theorem 1 to construct on $M$ a torsionfree affine connection with holonomy in $G_{3}$. Any such connection can be constructed, at least locally, along these lines, and how this works in practice will be shown in the next subsection.

3.3. An explicit torsion-free affine connection with holonomy $G_{3}$ on Pedersen's moduli space. In this subsection we apply the machinery developed above to a particular twistor space $Y=\mathrm{C}\left(\Omega^{1} Z\right)$, where $Z$ is a point blow-up of the 2-fold covering of a neighbourhood of a $(1,4)$ curve [Ped] in a quadric $\mathrm{CP}^{1} \times \mathrm{CP}^{1}$, branched along the curve. According to [Ped], this $Z$ contains a rational curve $X$ with self-intersection number 3 and the associated Kodaira's complete family of rational curves admits a very simple explicit description. The projectivised conormal bundle $\hat{X}=\mathrm{P}\left(N^{*}\right)$ of $X$ in $Z$ is itself a 
projective line naturally embedded into $Y=\mathrm{P}\left(\Omega^{1} Z\right)$ as a Legendre submanifold with $L_{\hat{X}}=\mathcal{O}(3)$. As was noted in subsection 2.1, the Kodaira moduli space of deformations of $X$ inside $Z$ is identical to the Legendre moduli space of deformations of $\hat{X}$ inside $Y$.

We start by considering $\mathrm{CP}^{1} \times \mathrm{CP}^{1}$, covered by the charts $(\eta, \rho) \cup(\hat{\eta}, \hat{\rho})$ : $\hat{\eta}=1 / \eta, \hat{\rho}=1 / \rho$. We can specify a curve $X$ of bidegree $(1,4)$ in $\mathrm{CP}^{1} \times \mathrm{CP}^{1}$ by putting $\eta=\rho^{4}$ and we change coordinates to $\left(\hat{u}=\hat{\rho}^{4}-\hat{\eta}, \hat{z}=\hat{\rho}\right) \cup$ $\left(u=\eta-\rho^{4}, z=\rho\right)$, so that $X$ is given by $u=0 . X$ has normal bundle $\mathcal{O}(8)$ in $C P^{1} \times C P^{1}$.

If $U$ is a two-to-one covering of a tubular neighbourhood of $X$ in $\mathrm{CP}^{1} \times \mathrm{CP}^{1}$ it has charts $(\hat{v}=\sqrt{\hat{u}}, \hat{z}) \cup(v=\sqrt{u}, z)$. Now $X$, which is given by $\hat{v}=v=0$, has normal bundle $\mathcal{O}(4)$ in $U$.

Finally, we blow up the point $v=z=0$ in $U$ to obtain our space $Z$ with charts $(\hat{w}=\hat{v}, \hat{z}) \cup(w=v / z, z)$, and patching function

$$
f(w, z)=\hat{w}=\frac{w}{z^{2} \sqrt{w^{2}+z^{2}}} .
$$

$Z$ contains our original curve $X$, which now has normal bundle $\mathcal{O}(3)$ as required.

We now seek a family of curves $X_{t}=\mathrm{CP}^{1} \in Z$ with normal bundle $\mathcal{O}(3)$. This family will project onto a family of $(1,4)$ curves in $\mathrm{CP}^{1} \times \mathrm{CP}^{1}$ meeting $X$ in four points to second order, one of these points being $u=z=0$. A general $(1,4)$ curve is given by $\eta=p(\rho) / q(\rho)$, for a pair of quartics $p$ and $q$. If we choose $p(z)=z^{2} P(z)$ and $q(z)=Q(z)$, then our condition can be written $z^{4} Q-z^{2} P=(z R)^{2}$, or

$$
P=-R^{2}+z^{2} Q
$$

for some cubic $R(z)$. Now let

$$
R(z)=t_{3} z^{3}+t_{2} z^{2}+t_{1} z+t_{0}
$$

and choose

$$
P(z)=z^{2}-2 t_{0} t_{1} z-t_{0}^{2}
$$

Then

$$
Q(z)=z^{2}\left(t_{3} z+t_{2}\right)^{2}+2\left(t_{3} z+t_{2}\right)\left(t_{1} z+t_{0}\right)+t_{1}^{2}+1 .
$$

The Legendre moduli space $M$ of $\mathrm{CP}^{1}$ 's in the contact three-fold $Y=\mathrm{P}_{Z}\left(\Omega^{1} Z\right)$ is equal to the Kodaira moduli space of $\mathrm{CP}^{1}$, $\mathrm{s}$ in $Z$. Let $t_{\alpha}$ be coordinates on $M$. Then $\forall t_{\alpha}$ the associated $X_{t}=\mathrm{CP}^{1} \in Z$ is given by

$$
w=\phi(z, t)=\frac{i R(z)}{\sqrt{Q(z)}}
$$


or

$$
\hat{w}=\hat{\phi}(\hat{z}, t)=\frac{i \hat{R}(\hat{z})}{\sqrt{\hat{P}(\hat{z})}}=\frac{i R(z)}{z^{2} \sqrt{P(z)}},
$$

where

$$
\begin{aligned}
& \hat{P}(\hat{z})=z^{-2} P(z) \\
& \hat{R}(\hat{z})=z^{-3} R(z) .
\end{aligned}
$$

How is this realised in $Y$ ? The charts are $(\hat{w}, \hat{u}, \hat{z}) \cup(w, u, z)$ and the contact one-form is $\hat{\Theta}=d \hat{w}+\hat{u} d \hat{z}, \Theta=d w+u d z$. The patching functions are

$$
\hat{w}=f(w, z), \hat{z}=1 / z,
$$

and

$$
\hat{u}=h(w, u, z)=\frac{\frac{\partial f}{\partial w} u-\frac{\partial f}{\partial z}}{\frac{\partial}{\partial z}(1 / z)},
$$

and $\forall t_{\alpha} \in M$ the associated Legendre curve $X_{t} \in Y$ is given by

$$
\begin{gathered}
w=\frac{i R(z)}{\sqrt{Q(z)}}, \quad u=-\frac{\partial \phi}{\partial z} \\
\hat{w}=\frac{i R(z)}{z^{2} \sqrt{P(z)}}, \quad \hat{u}=-\frac{\partial \hat{\phi}}{\partial \hat{z}} .
\end{gathered}
$$

The contact line bundle $L$ has patching function given by $\hat{\Theta}=A \Theta$, where

$$
A=\frac{\partial f}{\partial w}=\left(w^{2}+z^{2}\right)^{-3 / 2}
$$

Define the bundle $E$ on $Y$ by the patching function $G=\left(w^{2}+z^{2}\right)^{-1 / 2}$. Then $L=E^{\otimes 3}$, as required.

Now we are ready to compute the connection. Since the patching function

$$
\left.G(w, z)\right|_{w=\phi(z, t)}=\frac{\sqrt{Q(z)}}{\sqrt{P(z)}}
$$

the bundle $\left.E\right|_{X_{t}}(=\mathcal{O}(1))$ has only two linearly independent global sections, given by

and

$$
\left\{\hat{\lambda}_{(0)}=\frac{\hat{z}}{\sqrt{\hat{P}}}, \quad \lambda_{(0)}=\frac{1}{\sqrt{Q}}\right\}
$$

$$
\left\{\hat{\lambda}_{(1)}=\frac{1}{\sqrt{\hat{P}}}, \quad \lambda_{(1)}=\frac{z}{\sqrt{Q}}\right\} .
$$


Equation (6) becomes

$$
\chi_{b A}=-\hat{\theta}_{c A} F_{b}^{c}+G \theta_{b A},
$$

where

$$
F_{b}^{c}=\left(\begin{array}{cc}
\frac{Q^{3 / 2}}{P^{3 / 2}} & 0 \\
\frac{3 z^{2} Q^{1 / 2}}{P^{1 / 2}} \frac{\partial}{\partial z}\left(\frac{Q}{P}\right) & \frac{-z^{2} Q^{3 / 2}}{P^{3 / 2}}
\end{array}\right)=\left(\begin{array}{cc}
\left.\frac{\partial f}{\partial w}\right|_{w=\phi(z, t)} & 0 \\
\frac{\partial h}{\partial w} & \frac{\partial h}{\partial u}
\end{array}\right) .
$$

$\chi_{b A}$ is given by

$$
\chi_{1 A}=\left.\frac{\partial G}{\partial w}\right|_{w=\phi(z, t)} \cdot \lambda_{A}
$$

and

$$
\chi_{2 A}=\left.\frac{\partial G}{\partial u}\right|_{w=\phi(z, t)} \cdot \lambda_{A}=0 .
$$

It is convenient to express the solution of equation (18) and the connection coefficients in terms of the two polynomials $\alpha$ and $\beta$ defined as follows. Let:

$$
a=1+t_{0} t_{2}+2 t_{1}^{2}, \quad b=t_{0}^{2} t_{3}-t_{1}, \quad \text { and } \quad \Delta=a^{2}-b^{2}+2 t_{1} a b .
$$

Then

$$
\alpha=a / 3 \Delta \text { and } \beta=b / 3 \Delta .
$$

The solution of equation (18) is

$$
\begin{gathered}
\hat{\theta}_{1(0)}=i \beta \hat{P}, \quad \hat{\theta}_{0(0)}=3 i t_{0}\left(\alpha+t_{1} \beta\right), \\
\hat{\theta}_{1(1)}=-i t_{0} \alpha \hat{P}, \quad \hat{\theta}_{0(1)}=3 i t_{0}^{2}\left(t_{1} \alpha-\beta\right) .
\end{gathered}
$$

At last, we can use this solution in equation (7) to calculate the connection coefficients, which are as follows.

$$
\begin{array}{ll}
\Gamma_{00}^{0}=2 \beta\left(t_{0} t_{3}+t_{1} t_{2}\right)-3 t_{0} t_{1} t_{2} \alpha & \Gamma_{00}^{1}=t_{1} t_{3} \beta \\
\Gamma_{01}^{0}=3 t_{1}^{2} \beta+\alpha\left(3 t_{1}+3 t_{0} t_{1}^{2}-2 t_{0} t_{1} t_{2}-2 t_{0}^{2} t_{3}\right) & \Gamma_{01}^{1}=-t_{0} t_{1} t_{3} \alpha \\
\Gamma_{10}^{0}=-4 t_{1}^{2} \beta-\alpha\left(2 t_{1}+t_{0}^{2} t_{3}\right) & \Gamma_{10}^{1}=t_{0} t_{3} \beta \\
\Gamma_{11}^{0}=3 t_{0} t_{1} \beta+t_{0} \alpha\left(1+t_{0} t_{2}\right) & \Gamma_{11}^{1}=-t_{0}^{2} t_{3} \alpha \\
& \\
\Gamma_{20}^{0}=-t_{0}\left(3 \alpha+4 t_{1} \beta\right) & \Gamma_{20}^{1}=\beta \\
\Gamma_{21}^{0}=t_{0}^{2}\left(3 \beta-2 t_{1} \alpha\right) & \Gamma_{21}^{1}=-t_{0} \alpha \\
& \\
\Gamma_{30}^{0}=t_{0}^{2} \beta & \Gamma_{30}^{1}=-t_{0}\left(3 \alpha+2 t_{1} \beta\right) \\
\Gamma_{31}^{0}=-t_{0}^{3} \alpha & \Gamma_{31}^{1}=t_{0}^{2}\left(3 \beta-4 t_{1} \alpha\right)
\end{array}
$$


With this explicit connection at hand it is a trivial matter to check that the curvature tensor of the spinor connection spans at a general point $t \in M$ the whole Lie algebra $\mathrm{gl}(2, \mathrm{C})$ which immediately implies that the associated torsion-free connection has holonomy group $G_{3}$ rather than one of its proper subgroups.

Acknowledgements. It is a pleasure to thank M. Eastwood, H. Pedersen and $\mathrm{P}$. Tod for valuable discussions.

\section{REFERENCES}

[Ar] V.I. Arnold, Mathematical Methods of Classical Mechanics, Springer-Verlag, New York, 1978 [Russian: Nauka, Moscow, 1974].

[B-E] R.J. Baston and M.G. Eastwood, The Penrose transform, its interaction with representation theory, Oxford University Press, Oxford, 1989.

[B] M. Berger, Sur les groupes d'holonomie des variétés á connexion affine et des variétés Riemanniennes, Bull. Soc. Math. France 83 (1955), 279-330.

[Br1] R. Bryant, Metrics with exceptional holonomy, Ann. of Math. (2) 126 (1987), 525-576.

[Br2] R. Bryant, Two exotic holonomies in dimension four, path geometries, and twistor theory, Proc. Sympos. Pure Math. 53 (1991), 33-88.

[E] M. Eastwood, The generalized Penrose-Ward transform, Math. Proc. Cambridge Philos. Soc. 97 (1985), 165-187.

[H-O] J. Hano and H. Ozeki, On the holonomy groups of linear connections, Nagoya Math. J. 10 (1956), 97-100.

[H] N. Hitchin, Complex manifolds and Einstein equations, in Lecture Notes in Math. 970 (1982), 73-99.

[K] K. Kodaira, A theorem of completeness of characteristic systems for analytic families of compact submanifolds of complex manifolds, Ann. of Math. 75 (1962), 146-162.

[L] J. Leiterer, The Penrose transform for bundles non-trivial on the general line Math. Nachr. 112 (1983), 33-67.

[M] Yu. I. Manin, Gauge Field theory and Complex Geometry, Springer-Verlag, 1988 [Russian: Nauka, Moscow, 1984].

[Me1] S. A. Merkulov, Existence and geometry of Legendre moduli spaces, Math. Z. 226 (1997), 211-265.

[Me2] S. A. Merkulov, Moduli of compact complex Legendre submanifolds of complex contact manifolds, Math. Res. Lett. 1 (1994), 717-727.

[Me3] S. A. Merkulov, Relative deformation theory and differential geometry, in Lecture Notes in Pure and Appl. Math. 169 (1995), 107-132.

[Ped] H. Pedersen, Einstein-Weyl spaces and $(1, n)$-curves in the quadric surface, Ann. Global Anal. Geom. 4 (1985), 89-120.

[Pen] R. Penrose, Non-linear gravitons and curved twistor theory, Gen. Rel. Grav. 7 (1976), 3152.

[W] R. Ward, On self-dual gauge fields, Phys. Lett. A 61 (1977), 81-82.

SCHOOL OF MATHEMATICS AND STATISTICS UNIVERSITY OF PLYMOUTH

PLYMOUTH, DEVON PL4 8AA UNITED KINGDOM
DEPARTMENT OF MATHEMATICS

GLASGOW UNIVERSITY

15 UNIVERSITY GARDENS

GLASGOW G12 8QW

UK 